\title{
Molecular titration promotes oscillations and bistability in minimal network models with monomeric regulators
}

\section{Supplementary Information}

Christian Cuba Samaniego ${ }^{1}$, Giulia Giordano ${ }^{2}$, Jongmin Kim³ ${ }^{3}$ Franco Blanchini ${ }^{2}$, and Elisa Franco ${ }^{1, *}$

${ }^{1}$ Department of Mechanical Engineering, University of California at Riverside, 900 University Avenue, Riverside, CA 92521, USA

${ }^{2}$ Department of Mathematics and Computer Science, University of Udine, via delle Scienze 206, 33100 Udine, Italy

${ }^{3}$ Wyss Institute for Biologically Inspired Engineering, Harvard University, 3 Blackfan Circle, Boston, MA 02115, USA

${ }^{*}$ Corresponding author 


\section{Contents}

1 Analysis of the inhibited and activated modules 3

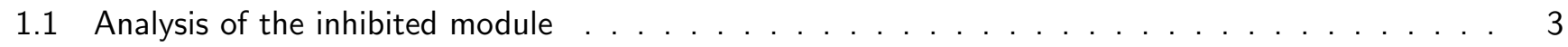

1.1.1 Analysis in the absence of direct titration reaction . . . . . . . . . . . . . . 3

1.1.2 Analysis in the presence of direct titration reactions . . . . . . . . . . . . . 5

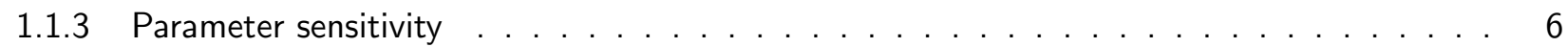

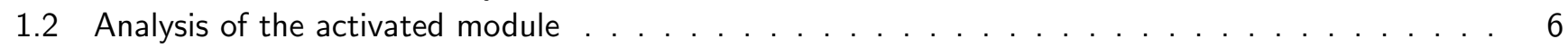

1.2.1 Analysis in the absence of direct titration reaction . . . . . . . . . . . . . . 6

1.2.2 Analysis in the presence of direct titration reactions . . . . . . . . . . . . . . . . . . . . . . . . . . . . .

1.2 .3 Parameter sensitivity . . . . . . . . . . . . . . . . . . 10

2 Oscillator $\quad 10$

2.1 Analysis in the absence of direct titration reactions . . . . . . . . . . . . . 11

2.1 .1 Equilibrium conditions . . . . . . . . . . . . . . . . . . . . . 11

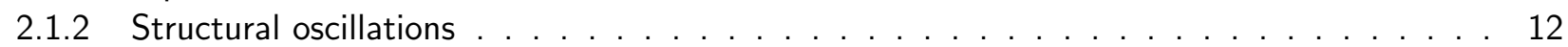

2.1 .3 Linear analysis . . . . . . . . . . . . . . . . . . . . . . . . 12

2.2 Analysis in the presence of direct titration reactions . . . . . . . . . . . . . . 13

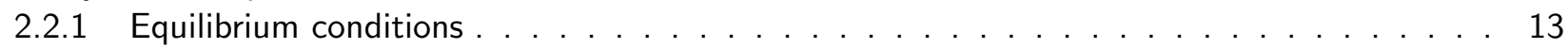

2.2 .2 Structural oscillations . . . . . . . . . . . . . . . . . . . . . . . . 14

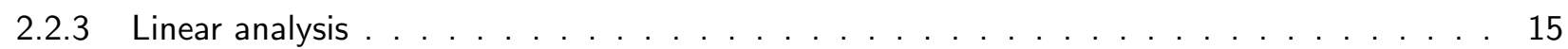

2.3 Numerical simulations . . . . . . . . . . . . . . . . . . . . . . . . 16

2.3.1 Randomized parameter search . . . . . . . . . . . . . . . . . . 16

2.3.2 Classification of dynamic behaviors in a region of the parameter space . . . . . . . . . 16

3 Bistable system $\quad 21$

3.1 Analysis in the absence of direct titration reactions . . . . . . . . . . . . . . 21

3.1 .1 Equilibrium conditions . . . . . . . . . . . . . . . . . . . . . 21

3.1 .2 Structural bistability . . . . . . . . . . . . . . . . . . . . . . 22

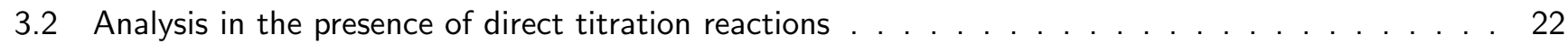

3.2 .1 Equilibrium conditions . . . . . . . . . . . . . . . . . . . . . 22

3.2 .2 Structural bistability . . . . . . . . . . . . . . . . . . . . . . . . 23

3.3 Numerical simulations . . . . . . . . . . . . . . . . . . . . . . . . 23

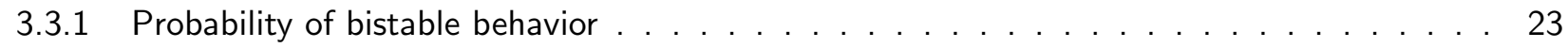

3.3.2 Bistable behavior in a region of the parameter space . . . . . . . . . . . . . . . 24 


\section{Analysis of the inhibited and activated modules}

The oscillator and the bistable systems considered in the main paper can be analyzed following the theory proposed in [1, 2], which allows us to show that they structurally have (by design) the capacity to respectively exhibit oscillations and bistability.

On the one hand, it can be shown that a system given by the feedback interconnection of an inhibitor module and an activator module is the negative feedback interconnection of two monotone systems, and is therefore a strong candidate oscillator according to [2] (if driven to instability, the system necessarily exhibits sustained oscillations). On the other hand, a system given by the feedback interconnection of mutually inhibiting modules is the positive feedback interconnection of two monotone systems, and is therefore a strong candidate bistable system according to [2] (if the system is driven to instability, bistable phenomena necessarily arise).

Here we demonstrate the same properties following an alternative, simplified route. We begin by analyzing the properties of the inhibited and the activated modules individually.

\subsection{Analysis of the inhibited module}

\subsubsection{Analysis in the absence of direct titration reaction}

We recall the model for the inhibited module:

$$
\begin{aligned}
\dot{x}_{T} & =\alpha\left(x_{T}^{t o t}-x_{T}\right) x_{A}-\delta x_{T} r_{I} \\
\dot{x}_{A} & =\kappa\left(x_{A}^{t o t}-x_{A}-x_{T}\right)-\alpha\left(x_{T}^{t o t}-x_{T}\right) x_{A} \\
\dot{r}_{I} & =\beta u_{I}-\delta x_{T} r_{I}-\phi r_{I} .
\end{aligned}
$$

Assumption 1. We assume that $x_{A}^{t o t} \geq x_{T}^{t o t}$.

Additionally, we make an assumption that relates parameters $\kappa, \alpha$, and the total concentrations of target and constitutive activator.

Assumption 2. We assume that $x_{A}^{t o t}-x_{T}^{t o t}-\frac{\kappa}{\alpha} \geq 0$.

Proposition 1. For a given concentration of inhibitor source $\bar{u}_{I}$, system (1)-(3) has unique equilibrium values $\bar{x}_{T}, \bar{x}_{A}$ and $\bar{r}_{I}$. The equilibrium $\bar{x}_{T}$ is a monotonic, strictly decreasing function of $\bar{u}_{I}$.

Proof. We first find an expression of $\bar{x}_{A}$ as a function of $\bar{x}_{T}$. From $\dot{x}_{A}=0$, we obtain:

$$
\bar{x}_{A}\left(\bar{x}_{T}\right)=\frac{\kappa\left(x_{A}^{t o t}-\bar{x}_{T}\right)}{\alpha\left(x_{T}^{t o t}-\bar{x}_{T}\right)+\kappa} \doteq g_{A}\left(\bar{x}_{T}\right)
$$

It can be verified that under Assumptions 1 and $2, \partial g_{A}\left(\bar{x}_{T}\right) / \partial \bar{x}_{T} \geq 0$. Thus $g_{A}\left(\bar{x}_{T}\right)$ is a continuous, monotonically increasing function of $\bar{x}_{T}$, and, for a given value of $u_{I}, \bar{x}_{T}$, there is a unique equilibrium $\bar{x}_{A}$.

Now we find $\bar{r}_{I}$ as a function of $\bar{x}_{T}$. From $\dot{x}_{T}=0$, We obtain:

$$
\begin{aligned}
\bar{r}_{I}\left(\bar{x}_{T}\right) & =\frac{\alpha\left(x_{T}^{t o t}-\bar{x}_{T}\right) \bar{x}_{A}\left(\bar{x}_{T}\right)}{\delta \bar{x}_{T}}=\frac{\alpha\left(x_{T}^{t o t}-\bar{x}_{T}\right)}{\delta \bar{x}_{T}} \frac{\kappa\left(x_{A}^{t o t}-\bar{x}_{T}\right)}{\alpha\left(x_{T}^{t o t}-\bar{x}_{T}\right)+\kappa} \\
& =\frac{\kappa}{\delta} \frac{\left(x_{A}^{t o t}-\bar{x}_{T}\right)}{\bar{x}_{T}} \frac{\left(x_{T}^{t o t}-\bar{x}_{T}\right)}{\left(x_{T}^{t o t}-\bar{x}_{T}\right)+\frac{\kappa}{\alpha}} \doteq \frac{\kappa}{\delta} A\left(\bar{x}_{T}\right) B\left(\bar{x}_{T}\right),
\end{aligned}
$$

where $A\left(\bar{x}_{T}\right) \doteq \frac{\left(x_{A}^{\text {tot }}-\bar{x}_{T}\right)}{\bar{x}_{T}}$ and $B\left(\bar{x}_{T}\right) \doteq \frac{\left(x_{T}^{\text {tot }}-\bar{x}_{T}\right)}{\left(x_{T}^{\text {to }}-\bar{x}_{T}\right)+\frac{\kappa}{\alpha}}$. Since $\partial A / \partial \bar{x}_{T}=-\frac{x_{A}^{\text {tot }}}{\bar{x}_{T}^{2}}<0, \partial B / \partial \bar{x}_{T}=-\frac{\frac{\kappa}{\alpha}}{\left(x_{T}^{\text {tot }}-\bar{x}_{T}+\frac{\kappa}{\alpha}\right)^{2}}<0$, and both $A\left(\bar{x}_{T}\right)$ and $B\left(\bar{x}_{T}\right)$ are positive for arbitrary parameter values (except at $\bar{x}_{T}=x_{T}^{\text {tot }}$, where $B\left(\bar{x}_{T}\right)=0$ ), 
we can conclude that the partial derivative $\partial \bar{r}_{I}\left(\bar{x}_{T}\right) / \partial \bar{x}_{T}=\frac{\kappa}{\delta}\left[\left(\partial A / \partial \bar{x}_{T}\right) B\left(\bar{x}_{T}\right)+A\left(\bar{x}_{T}\right)\left(\partial B / \partial \bar{x}_{T}\right)\right]<0$, thus $\bar{r}_{I}$ is a monotonic, strictly decreasing function of $\bar{x}_{T}$.

Finally, from $\dot{r}_{I}=0$ we find

$$
\bar{u}_{I}=\frac{1}{\beta}\left(\delta \bar{x}_{T}+\phi\right) \bar{r}_{I}\left(\bar{x}_{T}\right) \doteq h\left(\bar{x}_{T}\right) .
$$

To verify that the introduced function $h\left(\bar{x}_{T}\right)$ is a strictly decreasing monotonic function of $\bar{x}_{T}$, we rewrite it as $h\left(\bar{x}_{T}\right)=\frac{1}{\beta}\left[\delta \bar{x}_{T} \bar{r}_{I}\left(\bar{x}_{T}\right)+\phi \bar{r}_{I}\left(\bar{x}_{T}\right)\right]=\frac{1}{\beta}\left[C\left(\bar{x}_{T}\right)+\phi r_{I}\left(\bar{x}_{T}\right)\right]$. Because $\beta$ and $\phi$ are positive constants, and we already verified that $\partial \bar{r}_{I}\left(\bar{x}_{T}\right) / \partial \bar{x}_{T}<0$, we only need to check that $\partial C\left(\bar{x}_{T}\right) / \partial \bar{x}_{T}<0$. It is sufficient to note that $C\left(\bar{x}_{T}\right)=\kappa\left(x_{A}^{t o t}-\bar{x}_{T}\right) B\left(\bar{x}_{T}\right)$ (see definition of $B\left(\bar{x}_{T}\right)$ above). Since $\left(\partial B / \partial \bar{x}_{T}\right)<0, \partial\left(x_{A}^{t o t}-\bar{x}_{T}\right) / \partial \bar{x}_{T}<0$, and both $B\left(\bar{x}_{T}\right)>0$ and $\left(x_{A}^{\text {tot }}-\bar{x}_{T}\right)>0$, we have that $\partial C\left(\bar{x}_{T}\right) / \partial \bar{x}_{T}<0$.

Being $h\left(\bar{x}_{T}\right)$ a continuous, monotonic, strictly deceasing function of $\bar{x}_{T}$, its inverse is also a continuous, monotonic, decreasing function: $\bar{x}_{T}=g\left(\bar{u}_{I}\right)=h^{-1}\left(\bar{u}_{I}\right)$. We conclude that the equilibrium $\bar{x}_{T}$ for a given $\bar{u}_{I}$ is unique, and so are the other equilibria $\bar{x}_{A}$ and $\bar{r}_{I}$. In particular, the higher the concentration of input $U_{I}$, the smaller the equilibrium concentration of $X_{T}$.

We can show that a suitable set is positively invariant for the system: namely, any trajectory starting in this set is confined in the set for all time instants.

Proposition 2. The set

$$
0 \leq x_{T} \leq x_{T}^{t o t}, \quad \frac{\kappa}{\alpha} \leq x_{A} \leq x_{A}^{t o t}, \quad r_{I} \geq 0
$$

is positively invariant [3] for the system (1)-(3) for any $u_{I}$.

Proof. Since all the variables are non-negative and the variables $x_{T}$ and $x_{A}$ cannot exceed their total values, all the constraints are obvious with the exception of $x_{A} \geq \kappa / \alpha$. We show that this constraint cannot be violated: if we start with $x_{A}(0) \geq \kappa / \alpha$, then the constraint is satisfied for all $t>0$. In fact, for $x_{A}=\kappa / \alpha$ we have

$$
\dot{x}_{A}=\kappa\left(x_{A}^{t o t}-\kappa / \alpha-x_{T}\right)-\alpha\left(x_{T}^{t o t}-x_{T}\right)(\kappa / \alpha)=\kappa\left(x_{A}^{t o t}-x_{T}^{t o t}-\kappa / \alpha\right) \geq 0
$$

due to Assumption 2.

Proposition 3. The solutions of system (1)-(3) are bounded.

Proof. Species $x_{T}$ and $x_{A}$ are bounded by assumption. The dynamics of the regulator satisfies the inequality $\dot{r}_{I}(t) \leq \beta u_{I}^{\max }-\phi r_{I}$. By applying the comparison principle [6], we conclude that

$$
r_{I}(t) \leq r_{I}(0) e^{-\phi t}+\beta u_{I}^{\max }\left(1-e^{-\phi t}\right) / \phi,
$$

which ensures $r_{I}(t) \leq \max \left\{r_{I}(0), \beta u_{I}^{\max } / \phi\right\}$ at any point in time.

Proposition 4. The unique equilibrium of system (1)-(3) is locally stable.

Proof. For a given input $u_{I}$, the system admits a unique equilibrium (see Proposition 1). The Jacobian matrix

$$
J_{I}=\left[\begin{array}{ccc}
-\left(\alpha \bar{x}_{A}+\delta \bar{r}_{I}\right) & \alpha\left(x_{T}^{\text {tot }}-\bar{x}_{T}\right) & -\delta \bar{x}_{T} \\
-\kappa+\alpha \bar{x}_{A} & -\left[\kappa+\alpha\left(x_{T}^{\text {tot }}-\bar{x}_{T}\right)\right] & 0 \\
-\delta \bar{r}_{I} & 0 & -\left(\delta \bar{x}_{T}+\phi\right)
\end{array}\right]
$$

can be recast as a Metzler matrix (namely, a matrix whose non-diagonal entries are nonnegative) by changing the sign to the last row and column; in fact term $\alpha \bar{x}_{A}-\kappa>0$ in view of Proposition 2. A Metzler matrix has exclusively eigenvalues with negative real part (hence, is stable) if and only if all the coefficients of its characteristic polynomial $\operatorname{det}\left(\lambda I-J_{I}\right)$ are positive. A computation of the characteristic polynomial of $J_{I}$ shows that all its coefficients are positive. 
Remark 1. Systems whose Jacobian is (or is similar, up to a change of sign, to) a Metzler matrix are called monotone. As we will show later, in the absence of titration both the oscillator and the bistable system can be seen as the interconnection of two monotone components (corresponding to the modules).

\subsubsection{Analysis in the presence of direct titration reactions}

When titration reactions are present, the equations describing the system become:

$$
\begin{aligned}
\dot{x}_{T} & =\alpha\left(x_{T}^{t o t}-x_{T}\right) x_{A}-\delta x_{T} r_{I} \\
\dot{x}_{A} & =\kappa\left(x_{A}^{t o t}-x_{A}-x_{T}\right)-\alpha\left(x_{T}^{t o t}-x_{T}\right) x_{A}-\nu x_{A} r_{I} \\
\dot{r}_{I} & =\beta u_{I}-\delta x_{T} r_{I}-\phi r_{I}-\nu x_{A} r_{I} .
\end{aligned}
$$

Proposition 5. The solutions of system (5)-(7) are globally bounded.

Proof. All of the variables are non-negative and the variables $x_{T}$ and $x_{A}$ are upper-bounded by their total values $x_{T}^{\text {tot }}$ and $x_{A}^{\text {tot }}$. The boundedness of $r_{I}$ can be proved resorting to the comparison principle, as previously done for the system in the absence of titration.

Equilibria: First we find an expression for $\bar{x}_{A}$ as a function of $\bar{x}_{T}$. From $\dot{x}_{T}+\dot{x}_{A}=0$, we obtain:

$$
\kappa\left(x_{A}^{t o t}-\bar{x}_{A}-\bar{x}_{T}\right)=\left(\delta \bar{x}_{T}+\nu \bar{x}_{A}\right) \bar{r}_{I},
$$

and from $\dot{x}_{T}=0$,

$$
\alpha\left(x_{T}^{t o t}-\bar{x}_{T}\right) \bar{x}_{A}=\delta \bar{x}_{T} \bar{r}_{I}
$$

Then

$$
\bar{r}_{I}=\frac{\alpha\left(x_{T}^{t o t}-\bar{x}_{T}\right) \bar{x}_{A}}{\delta \bar{x}_{T}}=\frac{\kappa\left(x_{A}^{t o t}-\bar{x}_{A}-\bar{x}_{T}\right)}{\delta \bar{x}_{T}+\nu \bar{x}_{A}} .
$$

We obtain a second order polynomial of the following form: $a_{x} \bar{x}_{A}^{2}+b_{x} \bar{x}_{A}+c_{x}=0$, where

$$
a_{x}=\frac{\alpha \nu\left(x_{T}^{t o t}-\bar{x}_{T}\right)}{\delta \bar{x}_{T}}, \quad b_{x}=\alpha\left(x_{T}^{t o t}-\bar{x}_{T}\right)+\kappa, \quad c_{x}=-\kappa\left(x_{A}^{t o t}-\bar{x}_{T}\right) .
$$

Since $c_{x}$ is always negative, there is a unique positive and acceptable solution:

$$
\bar{x}_{A}\left(\bar{x}_{T}\right)=\frac{-b_{x}+\sqrt{b_{x}^{2}-4 a_{x} c_{x}}}{2 a_{x}} .
$$

With this expression we can find $\bar{r}_{I}$ as a function of $\bar{x}_{T}$. From $\dot{x}_{T}=0$, we obtain:

$$
\bar{r}_{I}\left(\bar{x}_{T}\right)=\frac{\alpha\left(x_{T}^{t o t}-\bar{x}_{T}\right) \bar{x}_{A}\left(\bar{x}_{T}\right)}{\delta \bar{x}_{T}} .
$$

From $\dot{r}_{I}=0$,

$$
\bar{u}_{I}=\frac{1}{\beta}\left(\delta \bar{x}_{T}+\phi+\nu \bar{x}_{A}\left(\bar{x}_{T}\right)\right) \bar{r}_{I}\left(\bar{x}_{T}\right) .
$$

Jacobian analysis: The Jacobian matrix becomes:

$$
J_{I}=\left[\begin{array}{ccc}
-\left(\alpha \bar{x}_{A}+\delta \bar{r}_{I}\right) & \alpha\left(x_{T}^{t o t}-\bar{x}_{T}\right) & -\delta \bar{x}_{T} \\
-\kappa+\alpha \bar{x}_{A} & -\left[\kappa+\alpha\left(x_{T}^{t o t}-\bar{x}_{T}\right)+\nu \bar{r}_{I}\right] & -\nu \bar{x}_{A} \\
-\delta \bar{r}_{I} & -\nu \bar{r}_{I} & -\left(\delta \bar{x}_{T}+\nu \bar{x}_{A}+\phi\right)
\end{array}\right]
$$


Proposition 6. The unique equilibrium of (5)-(7) is locally stable.

Proof. Let $p(s)=\operatorname{det}\left(s I-J_{I}\right)=p_{3} s^{3}+p_{2} s^{2}+p_{1} s+p_{0}$ be the characteristic polynomial of the linearized system. The polynomial is:

$$
p(s)=\operatorname{det}\left[\begin{array}{ccc}
s+(a+b) & -c & d \\
\kappa-a & s+(\kappa+c+n) & m \\
b & n & s+(d+m+\phi)
\end{array}\right] .
$$

We have $p_{3}=1, p_{2}=a+b+c+d+n+m+\phi+\kappa$,

$$
p_{1}=\operatorname{det}\left[\begin{array}{cc}
-(a+b) & c \\
a-\kappa & -(\kappa+c+n)
\end{array}\right]+\operatorname{det}\left[\begin{array}{cc}
-(a+b) & -d \\
-b & -(d+m+\phi)
\end{array}\right]+\operatorname{det}\left[\begin{array}{cc}
-(\kappa+c+n) & -m \\
-n & -(d+m+\phi)
\end{array}\right] \text {, }
$$

and finally $p_{0}=\operatorname{det}\left(-J_{I}\right)$. Some simple and tedious computations show that $p_{k}>0, k=0,1,2,3$. This is necessary, yet not sufficient for stability. According to the Routh-Hurwitz criterion, a polynomial has roots with negative real part if and only if the elements of the first column of the Routh-Hurwitz table are positive. Such a table is:

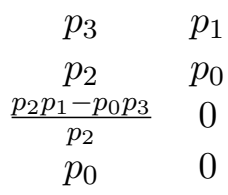

Then $p_{3}=1>0, p_{2}>0$ and $p_{0}>0$. It can be verified analytically that also $p_{2} p_{1}-p_{0} p_{3}>0$, for all positive values of the coefficients.

\subsubsection{Parameter sensitivity}

We numerically solved the ODEs describing the behavior of the inhibited module when each reaction rate is varied in a range. ODEs were integrated using MATLAB ode23s routine. The results are shown in Fig. S1.

\subsection{Analysis of the activated module}

\subsubsection{Analysis in the absence of direct titration reaction}

We recall the model for the activated module:

$$
\begin{aligned}
\dot{x}_{T} & =\alpha\left(x_{T}^{t o t}-x_{T}\right) r_{A}-\delta x_{T} x_{I} \\
\dot{x}_{I} & =\kappa\left(x_{I}^{t o t}-x_{I}-\left(x_{T}^{t o t}-x_{T}\right)\right)-\delta x_{T} x_{I} \\
\dot{r}_{A} & =\beta u_{A}-\alpha\left(x_{T}^{t o t}-x_{T}\right) r_{A}-\phi r_{A}
\end{aligned}
$$

Assumption 3. We assume that $x_{I}^{t o t} \geq x_{T}^{t o t}$.

Additionally, we make an assumption that relates parameters $\kappa, \delta$, and the total concentrations of target and constitutive inhibitor.

Assumption 4. We assume that $x_{I}^{t o t}-x_{T}^{t o t}-\frac{\kappa}{\delta} \geq 0$.

Proposition 7. For a given concentration of activator source $\bar{u}_{A}$, system (8)-(10) has unique equilibrium values $\bar{x}_{T}, \bar{x}_{I}$ and $\bar{r}_{A}$. The equilibrium $\bar{x}_{T}$ is a monotonic, strictly increasing function of $\bar{u}_{A}$. 

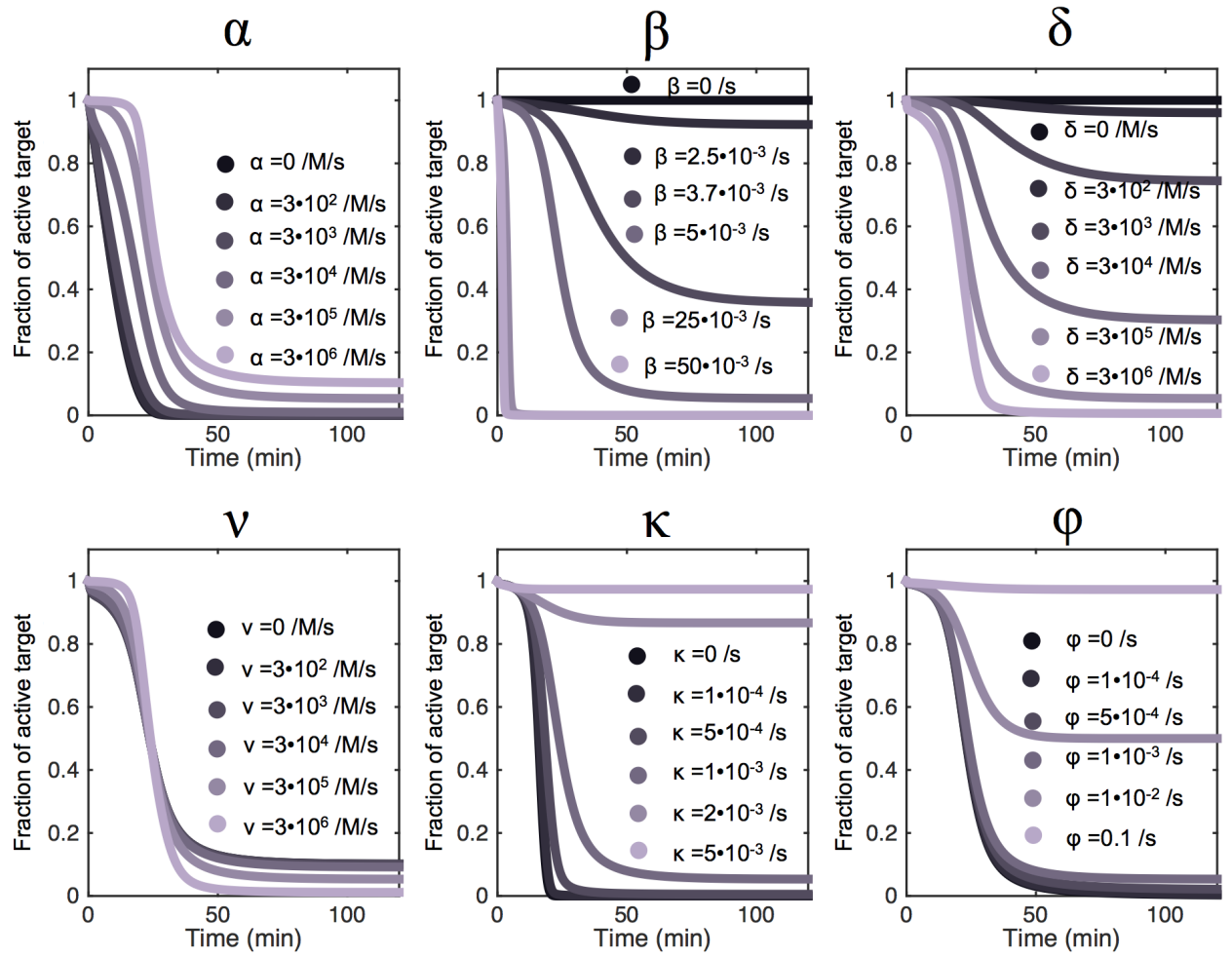

Figure S1: Numerical simulation showing the dependence of the normalized target concentration of the inhibited module $\left(x_{T}(t) / x_{T}^{t o t}\right)$ when the reaction rates are varied. 
Proof. We begin by finding an expression for $\bar{x}_{I}$ as a function of $\bar{x}_{T}$. From $\dot{x}_{I}=0$, we obtain:

$$
\bar{x}_{I}\left(\bar{x}_{T}\right)=\frac{\kappa\left(x_{I}^{t o t}-\left(x_{T}^{t o t}-\bar{x}_{T}\right)\right)}{\delta \bar{x}_{T}+\kappa} .
$$

The equilibrium $\bar{x}_{I}$ is a monotonic decreasing function of $\bar{x}_{T}$, as can be seen by checking the sign of the partial derivative:

$$
\frac{\partial \bar{x}_{I}}{\partial \bar{x}_{T}}=-\frac{\delta}{\kappa} \frac{x_{I}^{t o t}-x_{T}^{t o t}-\frac{\kappa}{\delta}}{\left(\frac{\delta}{\kappa} \bar{x}_{T}+1\right)^{2}} \leq 0,
$$

in view of Assumption 4.

We continue by finding $\bar{r}_{A}$ as a function of $\bar{x}_{T}$. From $\dot{x}_{T}=0$, we obtain:

$$
\bar{r}_{A}\left(\bar{x}_{T}\right)=\frac{\delta \bar{x}_{T}}{\alpha\left(x_{T}^{t o t}-\bar{x}_{T}\right)} \bar{x}_{I}\left(\bar{x}_{T}\right)
$$

With the same approach followed in the proof of Proposition 1, we can show that the equilibrium $\bar{r}_{A}$ is a monotonic, strictly increasing function of $\bar{x}_{T}$. Finally, from $\dot{r}_{A}=0$ we find:

$$
\bar{u}_{A}=\frac{1}{\beta}\left(\alpha\left(x_{T}^{t o t}-\bar{x}_{T}\right)+\phi\right) \bar{r}_{A}\left(\bar{x}_{T}\right)=\frac{1}{\beta}\left(\delta \bar{x}_{T} \bar{x}_{I}\left(\bar{x}_{T}\right)+\phi \bar{r}_{A}\left(\bar{x}_{T}\right)\right) \doteq w\left(\bar{x}_{T}\right) .
$$

To identify structural trends between $\bar{u}_{A}$ and $\bar{x}_{T}$, we check the sign of the partial derivative $\partial\left(\bar{x}_{T} \bar{x}_{I}\left(\bar{x}_{T}\right)\right) / \partial \bar{x}_{T}$; some tedious computations show that this partial derivative is always strictly positive, except for $\bar{x}_{T}=0$. Therefore $w\left(\bar{x}_{T}\right)$ is a monotonic, strictly increasing function of $\bar{x}_{T}$; its inverse $\bar{x}_{T} \doteq k\left(\bar{u}_{I}\right)=w^{-1}\left(\bar{u}_{I}\right)$ is thus also a strictly increasing function. We conclude that the equilibrium $\bar{x}_{T}$ for a given $\bar{u}_{A}$ is unique, and so are the other equilibria $\bar{x}_{I}$ and $\bar{r}_{A}$. In particular, the higher the concentration of input $U_{A}$, the smaller the equilibrium concentration of $X_{T}$.

Proposition 8. The set

$$
0 \leq x_{T} \leq x_{T}^{t o t}, \quad \frac{\kappa}{\delta} \leq x_{I} \leq x_{I}^{t o t}, \quad r_{A} \geq 0
$$

is positively invariant [3] for (8)-(10).

Proof. Since all the variables are non-negative and the variables $x_{T}$ and $x_{I}$ cannot exceed their total values, all the constraints are obvious with the exception of $\kappa / \delta \leq x_{I}$. We show that this constraint cannot be violated: if we start with $x_{I}(0) \geq \kappa / \delta$, then the constraint is satisfied for all $t>0$. In fact, due to Assumption 4 , for $x_{I}=\kappa / \delta$ we have

$$
\dot{x}_{I}=\kappa\left(x_{I}^{t o t}-\frac{\kappa}{\delta}-x_{T}^{t o t}+x_{T}\right)-\delta x_{T} \frac{\kappa}{\delta}=\kappa\left(x_{I}^{t o t}-x_{T}^{t o t}-\frac{\kappa}{\delta}\right) \geq 0 .
$$

Proposition 9. The solutions of system (8)-(10) are bounded.

Proof. Species $x_{T}$ and $x_{I}$ are bounded by assumption. The dynamics of the regulator satisfies the inequality $\dot{r}_{A}(t) \leq \beta u_{A}^{\max }-\phi r_{A}$. In view of the comparison principle, $r_{A}(t) \leq r_{A}(0) e^{-\phi t}+u_{A}^{\max } \beta\left(1-e^{-\phi t}\right) / \phi$, which ensures $r_{A}(t) \leq \max \left\{r_{A}(0), u_{A}^{\max } \beta / \phi\right\}$ at any point in time.

Proposition 10. The unique equilibrium of system (8)-(10) is locally stable. 
Proof. We follow the proof of Proposition 4. The Jacobian matrix is

$$
J_{A}=\left[\begin{array}{ccc}
-\left(\alpha \bar{r}_{A}+\delta \bar{x}_{I}\right) & -\delta x_{T} & \alpha\left(x_{T}^{t o t}-\bar{x}_{T}\right) \\
\kappa-\delta \bar{x}_{I} & -\left(\kappa+\delta \bar{x}_{T}\right) & 0 \\
\alpha \bar{r}_{A} & 0 & -\alpha\left(x_{T}^{t o t}-\bar{x}_{T}\right)-\phi
\end{array}\right]
$$

and, since $\delta \bar{x}_{I}-\kappa>0$ (Proposition 8), it can be recast as a Metzler matrix by changing sign to its second row and column. As can be shown by direct computation, all the coefficients of the characteristic polynomial $\operatorname{det}\left(\lambda I-J_{A}\right)$ are positive.

\subsubsection{Analysis in the presence of direct titration reactions}

When titration reactions are present, the model becomes:

$$
\begin{aligned}
\dot{x}_{T} & =\alpha\left(x_{T}^{t o t}-x_{T}\right) r_{A}-\delta x_{T} x_{I} \\
\dot{x}_{I} & =\kappa\left(x_{I}^{t o t}-x_{I}-\left(x_{T}^{t o t}-x_{T}\right)\right)-\delta x_{T} x_{I}-\nu x_{I} r_{A} \\
\dot{r}_{A} & =\beta u_{A}-\alpha\left(x_{T}^{t o t}-x_{T}\right) r_{A}-\phi r_{A}-\nu x_{I} r_{A}
\end{aligned}
$$

Proposition 11. The solutions of system (12)-(14) are globally bounded.

Proof. Analogous to that of Proposition 5.

Equilibria: First we find an expression for $\bar{x}_{I}$ as a function of $\bar{x}_{T}$. From $\dot{x}_{T}-\dot{x}_{I}=0$, we obtain:

$$
\kappa\left(x_{I}^{t o t}-\bar{x}_{I}-\left(x_{T}^{t o t}-\bar{x}_{T}\right)\right)=\left(\alpha\left(x_{T}^{t o t}-\bar{x}_{T}\right)+\nu \bar{x}_{I}\right) \bar{r}_{A},
$$

and from $\dot{x}_{T}=0$,

$$
\alpha\left(x_{T}^{t o t}-\bar{x}_{T}\right) \bar{r}_{A}=\delta \bar{x}_{T} \bar{x}_{I}
$$

Then

$$
\bar{r}_{A}=\frac{\kappa\left(x_{I}^{t o t}-\bar{x}_{I}-\left(x_{T}^{t o t}-\bar{x}_{T}\right)\right)}{\alpha\left(x_{T}^{t o t}-\bar{x}_{T}\right)+\nu \bar{x}_{I}}=\frac{\delta \bar{x}_{T} \bar{x}_{I}}{\alpha\left(x_{T}^{t o t}-\bar{x}_{T}\right)}
$$

and we obtain a second order polynomial of the following form: $a_{x} \bar{x}_{I}^{2}+b_{x} \bar{x}_{I}+c_{x}=0$, where

$$
a_{x}=\frac{\delta \nu \bar{x}_{T}}{\alpha\left(x_{T}^{t o t}-\bar{x}_{T}\right)}, \quad b_{x}=\alpha \bar{x}_{T}+\kappa, \quad c_{x}=-\kappa\left(x_{I}^{t o t}-\left(x_{T}^{t o t}-\bar{x}_{T}\right)\right) .
$$

Since $c_{x}$ is always negative, the unique positive and acceptable solution is

$$
\bar{x}_{I}\left(\bar{x}_{T}\right)=\frac{-b_{x}+\sqrt{b_{x}^{2}-4 a_{x} c_{x}}}{2 a_{x}} .
$$

Then, we find $\bar{r}_{A}$ as a function of $\bar{x}_{T}$. From $\dot{x}_{T}=0$, we obtain:

$$
\bar{r}_{A}\left(\bar{x}_{T}\right)=\frac{\delta \bar{x}_{T} \bar{x}_{I}\left(\bar{x}_{T}\right)}{\alpha\left(x_{T}^{t o t}-\bar{x}_{T}\right)} .
$$

Finally from $\dot{r}_{A}=0$ :

$$
\bar{u}_{A}=\frac{1}{\beta}\left(\alpha\left(x_{T}^{t o t}-\bar{x}_{T}\right)+\phi+\nu \bar{x}_{I}\left(\bar{x}_{T}\right)\right) \bar{r}_{A}\left(\bar{x}_{T}\right) .
$$

Jacobian analysis: The Jacobian matrix is now:

$$
J_{A}=\left[\begin{array}{ccc}
-\left(\alpha \bar{r}_{A}+\delta \bar{x}_{I}\right) & -\delta \bar{x}_{T} & \alpha\left(x_{T}^{\text {tot }}-\bar{x}_{T}\right) \\
\kappa-\delta \bar{x}_{I} & -\left(\kappa+\delta \bar{x}_{T}+\nu \bar{r}_{A}\right) & -\nu \bar{x}_{I} \\
\alpha \bar{r}_{A} & -\nu \bar{r}_{A} & -\left(\alpha\left(x_{T}^{t o t}-\bar{x}_{T}\right)+\nu \bar{x}_{I}+\phi\right)
\end{array}\right]
$$


Proposition 12. The unique equilibrium of (12)-(14) is locally stable.

Proof. Analogous to that of Proposition 6.

\subsubsection{Parameter sensitivity}

Fig. S2 shows the behavior of the activated module when each reaction rates is varied in a range. ODEs were integrated using MATLAB ode23s routine.
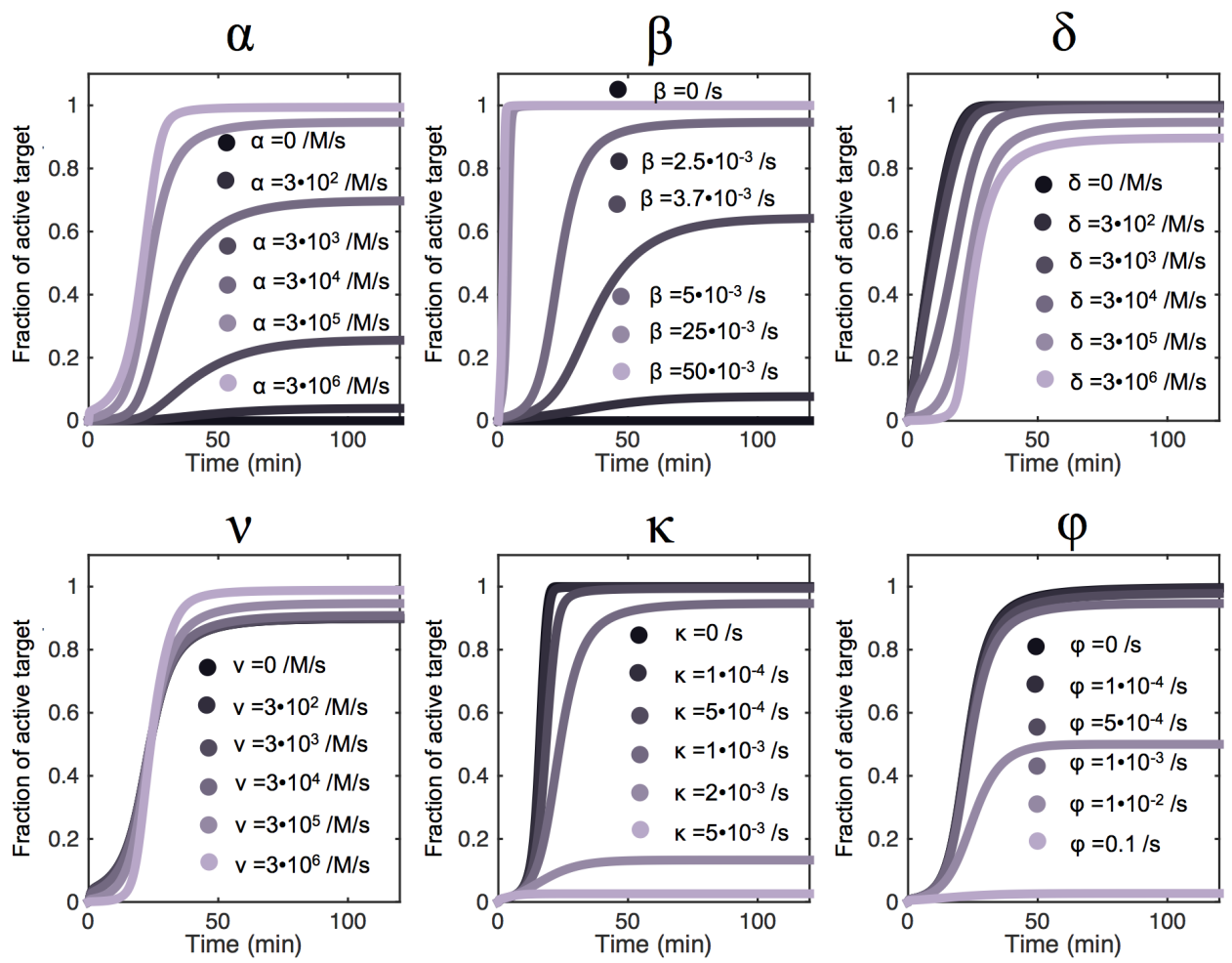

Figure S2: Numerical simulation showing the dependence of the normalized target concentration of the activated module $\left(x_{T}(t) / x_{T}^{t o t}\right)$ when the reaction rates are varied.

\section{Oscillator}

As discussed in the main text, we build an oscillator via the feedback interconnection of an inhibited module and an activated module. The reactions occurring in the system are: 


\section{Activated subsystem}

Activation:

Output production:

Inhibition:

Conversion:

Direct titration:

Degradation:

$$
X_{R, A}+Z_{T}^{*} \stackrel{\alpha_{z}}{\longrightarrow} Z_{T}+Z_{I}^{*}
$$$$
Z_{T} \stackrel{\beta_{z}}{\longrightarrow} Z_{R, I}+Z_{T}
$$$$
Z_{T}+Z_{I} \stackrel{\delta_{z}}{\longrightarrow} Z_{T}^{*}
$$$$
Z_{I}^{*} \stackrel{\kappa_{z}}{\longrightarrow} Z_{I}
$$$$
X_{R, A}+Z_{I} \stackrel{\nu_{z}}{\longrightarrow} Z_{I}^{*}
$$$$
Z_{R, I} \stackrel{\phi_{z}}{\longrightarrow} 0
$$

\section{Inhibited subsystem}

$$
\begin{aligned}
& X_{A}+X_{T}^{*} \stackrel{\alpha_{x}}{\longrightarrow} X_{T} \\
& X_{T} \stackrel{\beta_{x}}{\longrightarrow} X_{R, A}+X_{T} \\
& Z_{R, I}+X_{T} \stackrel{\delta_{x}}{\longrightarrow} X_{T}^{*}+X_{A}^{*} \\
& X_{A}^{*} \stackrel{\kappa_{x}}{\longrightarrow} X_{A} \\
& Z_{R, I}+X_{A} \stackrel{\nu_{x}}{\longrightarrow} X_{A}^{*} . \\
& X_{R, A} \stackrel{\phi_{x}}{\longrightarrow} 0
\end{aligned}
$$

The regulators interconnecting the modules are $x_{R, A}$, which is the output of the inhibited module and works as an activator for the activated module, and $z_{R, I}$, which is the output of the activated module and works as an inhibitor for the inhibited module. We recall that we assume mass conservation for species $Z_{T}, Z_{I}, X_{T}$, and $X_{A}$ : $z_{T}^{\text {tot }}=z_{T}+z_{T}^{*}, z_{I}^{\text {tot }}=z_{I}+z_{I}^{*}+z_{T}^{*}, x_{T}^{\text {tot }}=x_{T}+x_{T}^{*}, x_{A}^{t o t}=x_{A}+x_{A}^{*}+x_{T}$. Using the law of mass action we derive the differential equations:

$$
\begin{aligned}
& \dot{z}_{T}=\alpha_{z}\left(z_{T}^{t o t}-z_{T}\right) x_{R, A}-\delta_{z} z_{T} z_{I}, \\
& \dot{z}_{I}=\kappa_{z}\left(z_{I}^{t o t}-z_{I}-\left(z_{T}^{t o t}-z_{T}\right)\right)-\delta_{z} z_{T} z_{I}-\nu_{z} x_{R, A} z_{I}, \\
& \dot{x}_{R, A}=\beta_{x} x_{T}-\alpha_{z}\left(z_{T}^{t o t}-z_{T}\right) x_{R, A}-\nu_{z} x_{R, A} z_{I}-\phi_{x} x_{R, A}, \\
& \dot{x}_{T}=\alpha_{x}\left(x_{T}^{t o t}-x_{T}\right) x_{A}-\delta_{x} x_{T} z_{R, I}, \\
& \dot{x}_{A}=\kappa_{x}\left(x_{A}^{t o t}-x_{A}-x_{T}\right)-\alpha_{x}\left(x_{T}^{t o t}-x_{T}\right) x_{A}-\nu_{x} x_{A} z_{R, I}, \\
& \dot{z}_{R, I}=\beta_{z} z_{T}-\delta_{x} x_{T} z_{R, I}-\nu_{x} x_{A} z_{R, I}-\phi_{z} z_{R, I} .
\end{aligned}
$$

Throughout our analysis, we assume that $z_{I}^{t o t} \geq z_{T}^{\text {tot }}$ and $x_{A}^{\text {tot }} \geq x_{T}^{\text {tot }}$.

As a preliminary result, we notice that the interconnection does not change the boundedness property of the solution.

Proposition 13. The solutions of system (15)-(20) are bounded.

Proof. The proposition follows from the fact that each subsystem has bounded solution for bounded inputs. Then we notice that the inhibited subsystem $\left(x_{T}-x_{A}-z_{R, I}\right)$ has input $\beta_{z} z_{T} \leq \beta_{z} z_{T}^{t o t}$, which is bounded, while the activated subsystem $\left(z_{T}-z_{I}-x_{R, A}\right)$ has input $\beta_{x} x_{T} \leq \beta_{x} x_{T}^{\text {tot }}$, bounded as well.

\subsection{Analysis in the absence of direct titration reactions}

\subsubsection{Equilibrium conditions}

In this section, we consider the oscillatory system (15)-(20) in the absence of titration reactions, i.e., with $\nu_{x}=\nu_{z}=0$. We derive equilibrium conditions that are consistent with those derived for the inhibited and activated module. First, we find an expression for $\bar{x}_{T}$ as a function of $\bar{z}_{T}$. From $\dot{z}_{I}=0$, we obtain:

$$
\bar{z}_{I}\left(\bar{z}_{T}\right)=\frac{\kappa_{z}\left(z_{I}^{t o t}-\left(z_{T}^{t o t}-\bar{z}_{T}\right)\right)}{\delta_{z} \bar{z}_{T}+\kappa_{z}} .
$$

From $\dot{z}_{T}=0$, we obtain:

$$
\bar{x}_{R, A}\left(\bar{z}_{T}\right)=\frac{\delta_{z} \bar{z}_{T} \bar{z}_{I}\left(\bar{z}_{T}\right)}{\alpha_{z}\left(z_{T}^{\text {tot }}-\bar{z}_{T}\right)} .
$$


From $\dot{x}_{R, A}=0$,

$$
\bar{x}_{T}=\frac{1}{\beta_{x}}\left(\alpha_{z}\left(z_{T}^{\text {tot }}-\bar{z}_{T}\right)+\phi_{x}\right) \bar{x}_{R, A}\left(\bar{z}_{T}\right) \doteq w\left(\bar{z}_{T}\right) .
$$

As shown in Proposition $1, \bar{x}_{T}$ is a monotonically decreasing function of $\bar{z}_{T}$.

We now find an expression for $\bar{z}_{T}$ as a function of $\bar{x}_{T}$. From $\dot{x}_{A}=0$, we obtain:

$$
\bar{x}_{A}\left(\bar{x}_{T}\right)=\frac{\kappa_{x}\left(x_{A}^{t o t}-\bar{x}_{T}\right)}{\alpha_{x}\left(x_{T}^{t o t}-\bar{x}_{T}\right)+\kappa_{x}} .
$$

From $\dot{x}_{T}=0$, we obtain:

From $\dot{z}_{R, I}=0$,

$$
\bar{z}_{R, I}\left(\bar{x}_{T}\right)=\frac{\alpha_{x}\left(x_{T}^{t o t}-\bar{x}_{T}\right) \bar{x}_{A}\left(\bar{x}_{T}\right)}{\delta_{x} \bar{x}_{T}}
$$

$$
\bar{z}_{T}=\frac{1}{\beta_{z}}\left(\delta_{x} \bar{x}_{T}+\phi_{z}\right) \bar{z}_{R, I}\left(\bar{x}_{T}\right) \doteq h\left(\bar{x}_{T}\right)
$$

As shown in Proposition 7, $\bar{z}_{T}$ is a monotonically increasing function of $\bar{x}_{T}$. The functions $h\left(\bar{x}_{T}\right)$ and $w\left(\bar{z}_{T}\right)$ found above, because of their opposite trend, can admit a single intersection in the plane $\left(\bar{x}_{T}, \bar{z}_{T}\right)$. These equilibrium conditions will be used to find numerically or graphically the unique equilibrium point of the system.

\subsubsection{Structural oscillations}

The equilibrium conditions derived earlier show that there exists a single equilibrium, around which we linearize the system. It is convenient to change the sign of some of the variables: $-z_{I},-x_{T},-x_{A}$. The Jacobian matrix of system (15)-(20) with $\nu_{z}=\nu_{x}=0$ becomes:

$$
J=\left[\begin{array}{ccc|ccc}
-\alpha_{z} \bar{x}_{R, A}-\delta_{z} \bar{z}_{I} & \delta_{z} \bar{z}_{T} & \alpha_{z}\left(z_{T}^{\text {tot }}-\bar{z}_{T}\right) & 0 & 0 & 0 \\
-\kappa_{z}+\delta_{z} \bar{z}_{I} & -\kappa_{z}-\delta_{z} \bar{z}_{T} & 0 & 0 & 0 & 0 \\
\alpha_{z} \bar{x}_{R, A} & 0 & -\alpha_{z}\left(z_{T}^{\text {tot }}-\bar{z}_{T}\right)-\phi_{x} & 0 & -\beta_{x} & 0 \\
\hline \beta_{z} & 0 & 0 & 0 & -\delta_{x} \bar{x}_{T}-\phi_{z} & \delta_{x} \bar{z}_{R, I} \\
0 & 0 & 0 & \delta_{x} \bar{x}_{T} & -\alpha_{x} \bar{x}_{A}-\delta_{x} \bar{z}_{R, I} & \alpha_{x}\left(x_{T}^{\text {tot }}-\bar{x}_{T}\right) \\
0 & 0 & 0 & 0 & -\kappa_{x}+\alpha_{x} \bar{x}_{A} & -\kappa_{x}-\alpha_{x}\left(x_{T}^{\text {tot }}-\bar{x}_{T}\right)
\end{array}\right]
$$

We call strong candidate oscillator [1, 2] a system which can be locally unstable exclusively due to the existence of complex conjugate eigenvalues with nonnegative real part (in other words, the system does not admit real nonnegative eigenvalues).

Proposition 14. Under Assumptions 1, 2, 3, and 4, system (15)-(20) is a strong candidate oscillator.

Proof. The computation of the characteristic polynomial $\operatorname{det}(\lambda I-J)$ reveals that all the coefficients are positive (note that we assume $-\kappa_{z}+\delta_{z} \bar{z}_{I}>0$ and $-\kappa_{x}+\alpha_{x} \bar{x}_{A}>0$ ). A polynomial with positive coefficients cannot have nonnegative real roots.

\subsubsection{Linear analysis}

The Jacobian (21) clearly shows that the system is the feedback interconnection of two subsystems of the third order. To simplify the notation we define:

$$
\begin{aligned}
a_{1} \doteq \delta_{z} \bar{z}_{T}, & a_{2} \doteq \delta_{x} \bar{x}_{T}, \\
b_{1} \doteq \delta_{z} \bar{z}_{I}, & b_{2} \doteq \delta_{x} \bar{z}_{R, I}, \\
c_{1} \doteq \alpha_{z} \bar{x}_{R, A}, & c_{2} \doteq \alpha_{x} \bar{x}_{A}, \\
d_{1} \doteq \alpha_{z}\left(z_{T}^{t o t}-\bar{z}_{T}\right), & d_{2} \doteq \alpha_{x}\left(x_{T}^{\text {tot }}-\bar{x}_{T}\right) \\
e_{1} \doteq \kappa_{z}, & e_{2} \doteq \kappa_{x}, \\
h_{1} \doteq \phi_{x}, & h_{2} \doteq \phi_{z},
\end{aligned}
$$


Then, defining vectors $\xi_{1}=\left[\begin{array}{lll}z_{T} & -z_{I} & x_{R, A}\end{array}\right]^{\top}$ and $\xi_{2}=\left[\begin{array}{lll}z_{R, I} & -x_{T} & -x_{A}\end{array}\right]^{\top}$, the linearized system can be rewritten as the feedback interconnection of:

$$
\dot{\xi}_{1}=A_{1} \xi_{1}+\beta_{x} B_{1} \omega_{2}, \quad \omega_{1}=C_{1} \xi_{1},
$$

and

$$
\dot{\xi}_{2}=A_{2} \xi_{2}+\beta_{z} B_{2} \omega_{1}, \quad \omega_{2}=C_{2} \xi_{2},
$$

where:

$$
A_{1}=\left[\begin{array}{ccc}
-\left(c_{1}+b_{1}\right) & a_{1} & d_{1} \\
-e_{1}+b_{1} & -\left(e_{1}+a_{1}\right) & 0 \\
c_{1} & 0 & -\left(d_{1}+h_{1}\right)
\end{array}\right], \quad B_{1}=\left[\begin{array}{c}
0 \\
0 \\
-1
\end{array}\right], \quad C_{1}=\left[\begin{array}{lll}
1 & 0 & 0
\end{array}\right]
$$

and

$$
A_{2}=\left[\begin{array}{ccc}
-\left(a_{2}+h_{2}\right) & b_{2} & 0 \\
a_{2} & -\left(c_{2}+b_{2}\right) & d_{2} \\
0 & -e_{2}+c_{2} & -\left(e_{2}+d_{2}\right)
\end{array}\right], \quad B_{2}=\left[\begin{array}{l}
1 \\
0 \\
0
\end{array}\right], \quad C_{2}=\left[\begin{array}{lll}
0 & 1 & 0
\end{array}\right] .
$$

By applying the Laplace transform method, we can obtain an input-output representation of the two subsystems in terms of their transfer functions ${ }^{1}: F_{1}(s)=-\frac{n_{1}(s)}{d_{1}(s)}$ and $F_{2}(s)=\frac{n_{2}(s)}{d_{2}(s)}$. Since the overall feedback loop is negative and all the coefficients of the numerator and denominator polynomials $n_{1}(s), d_{1}(s), n_{2}(s), d_{2}(s)$ are positive, the closed-loop characteristic polynomial also has positive coefficients. Therefore it cannot admit non-negative real roots, as stated in the following proposition, hence the system is a strong candidate oscillator.

Proposition 15. Consider system (15)-(20), where $\nu_{x}=\nu_{z}=0$, linearized around its only equlibrium point. Its characteristic polynomial has no real nonnegative roots. If instability occurs, it is oscillatory, namely due to complex roots with positive real part.

The fact that the system is a candidate oscillator in the strong sense does not mean that the system oscillates for any choice of the parameters. In fact, numerical simulations show that oscillations occur only in a limited region in the plane defined by $\beta_{z}$ and $\beta_{x}$.

It is worth noticing that in the absence of titration reactions, being $c_{2}-e_{2}$ and $b_{1}-e_{1}$ positive quantities, the system is the negative feedback interconnection of two monotone subsystems, associated with the modules (see $[1,2]$ and the references therein): this structurally explains its oscillatory nature.

\subsection{Analysis in the presence of direct titration reactions}

\subsubsection{Equilibrium conditions}

We consider in this section the oscillatory system (15)-(20) in the presence of non-zero $\nu_{x}$ and $\nu_{z}$. We begin by finding two expressions of $\bar{x}_{R, A}$ as a function of the other variables. This can be done by setting $\dot{z}_{T}-\dot{z}_{I}=0$ and $\dot{z}_{T}=0$. Then, we equate the two expressions for $\bar{x}_{R, A}$ and we achieve:

$$
\frac{\delta_{z} \bar{z}_{T} \bar{z}_{I}}{\alpha_{z}\left(z_{T}^{t o t}-\bar{z}_{T}\right)}=\frac{\kappa_{z}\left(z_{I}^{t o t}-\bar{z}_{I}-\left(z_{T}^{t o t}-\bar{z}_{T}\right)\right)}{\alpha_{z}\left(z_{T}^{t o t}-\bar{z}_{T}\right)+\nu_{z} \bar{z}_{I}}
$$

\footnotetext{
${ }^{1}$ Given a linear system with an input $u(t)$ and an output $y(t)$, its transfer function $F(s)$ is the ratio between the Laplace transform of the output and the Laplace transform of the input: $F(s)=\frac{Y(s)}{U(s)}$.
} 
which defines a relationship between $\bar{z}_{T}$ and $\bar{z}_{I}$ at steady state. The equilibrium $\bar{z}_{I}$ can thus be derived as the solution of the second order equation $a_{z} \bar{z}_{I}^{2}+b_{z} \bar{z}_{I}+c_{z}=0$, where $a_{z}=\left(\frac{\delta_{z} \nu_{z}}{\alpha_{z}}\right) \frac{\bar{z}_{T}}{z_{T}^{\text {tot }}-\bar{z}_{T}}, \quad b_{z}=\left(\delta_{z} \bar{z}_{T}+\kappa_{z}\right)$ and $c_{z}=-\kappa_{z}\left(z_{I}^{t o t}-\left(z_{T}^{t o t}-\bar{z}_{T}\right)\right)$. Assuming $z_{I}^{\text {tot }}>z_{T}^{\text {tot }}$, since $a_{z} c_{z}<0$, the only admissible positive solution is:

$$
\bar{z}_{I}\left(\bar{z}_{T}\right)=\frac{-b_{z}+\sqrt{b_{z}^{2}-4 a_{z} c_{z}}}{2 a_{z}} .
$$

Then,

$$
\bar{x}_{R, A}=\frac{\delta_{z} \bar{z}_{T} \bar{z}_{I}\left(\bar{z}_{T}\right)}{\alpha_{z}\left(z_{T}^{t o t}-\bar{z}_{T}\right)} .
$$

Finally, we can find $\bar{x}_{T}$ as a function of $\bar{z}_{T}$, by setting $\dot{x}_{R, A}=0$ :

$$
\bar{x}_{T}=\frac{1}{\beta_{x}}\left(\alpha_{z}\left(z_{T}^{\text {tot }}-\bar{z}_{T}\right)+\phi_{x}+\nu_{z} \bar{z}_{I}\left(\bar{z}_{T}\right)\right) \bar{x}_{R, A}\left(\bar{z}_{T}\right) .
$$

We can proceed similarly to derive $\bar{z}_{T}$ as a function of $\bar{x}_{T}$. Setting $\dot{x}_{T}+\dot{x}_{A}=0$ and $\dot{x}_{T}=0$, we find two different expressions for $\bar{z}_{R, I}$. Equating these expressions we find:

$$
\frac{\alpha_{x}\left(x_{T}^{t o t}-\bar{x}_{T}\right) \bar{x}_{A}}{\delta_{x} \bar{x}_{T}}=\frac{\kappa_{x}\left(x_{A}^{t o t}-\bar{x}_{A}-\bar{x}_{T}\right)}{\delta_{x} \bar{x}_{T}+\nu_{x} \bar{x}_{A}}
$$

so we can isolate the relationship between $\bar{x}_{T}$ and $\bar{x}_{A}$ at steady state. As done before, we derive the equilibrium $\bar{x}_{A}$ as the solution of the second order equation $a_{x} \bar{x}_{A}^{2}+b_{x} \bar{x}_{A}+c_{x}=0$, where $a_{x}=\left(\frac{\alpha_{x} \nu_{x}}{\delta_{x}}\right) \frac{x_{T}^{\text {tot }}-\bar{x}_{T}}{\bar{x}_{T}}$, $b_{x}=\left(\alpha_{x}\left(x_{T}^{t o t}-\bar{x}_{T}\right)+\kappa_{x}\right)$ and $c_{x}=-\kappa_{x}\left(x_{A}^{t o t}-\bar{x}_{T}\right)$. Assuming $x_{A}^{\text {tot }}>x_{T}^{\text {tot }}$, since again $a_{x} c_{x}<0$, the only admissible positive solution is:

$$
\bar{x}_{A}\left(\bar{x}_{T}\right)=\frac{-b_{x}+\sqrt{b_{x}^{2}-4 a_{x} c_{x}}}{2 a_{x}} .
$$

Then,

$$
\bar{z}_{R, I}=\frac{\alpha_{x}\left(x_{T}^{t o t}-\bar{x}_{T}\right) \bar{x}_{A}\left(\bar{x}_{T}\right)}{\delta_{x} \bar{x}_{T}} .
$$

Finally, we can find $\bar{z}_{T}$ as a function of $\bar{x}_{T}$, by setting $\dot{z}_{R, I}=0$ :

$$
\bar{z}_{T}=\frac{1}{\beta_{z}}\left(\delta_{x} \bar{x}_{T}+\phi_{z}+\nu_{x} \bar{x}_{A}\left(\bar{x}_{T}\right)\right) \bar{z}_{R, I}\left(\bar{x}_{T}\right) .
$$

Once we find the only admissible equilibrium values $\bar{z}_{T}, \bar{z}_{I}, \bar{x}_{T}$ and $\bar{x}_{A}$ we can find $\bar{z}_{R, I}$ and $\bar{x}_{R, A}$.

$$
\begin{aligned}
& \dot{z}_{R, I}=0 \Longrightarrow \quad \bar{z}_{R, I}=\frac{\beta_{z} \bar{z}_{T}}{\delta_{x} \bar{x}_{T}+\nu_{x} \bar{x}_{A}+\phi_{z}}, \\
& \dot{x}_{R, A}=0 \quad \Longrightarrow \quad \bar{x}_{R, A}=\frac{\beta_{x} \bar{x}_{T}}{\alpha_{z}\left(z_{T}^{\text {tot }}-\bar{z}_{T}\right)+\nu_{z} \bar{z}_{I}+\phi_{x}} .
\end{aligned}
$$

\subsubsection{Structural oscillations}

As done before, we change the sign to some of the variables, which become $-z_{I},-x_{T},-x_{A}$; the Jacobian of the system in the presence of titration reactions becomes matrix $J_{\nu}$

$J_{\nu}=$

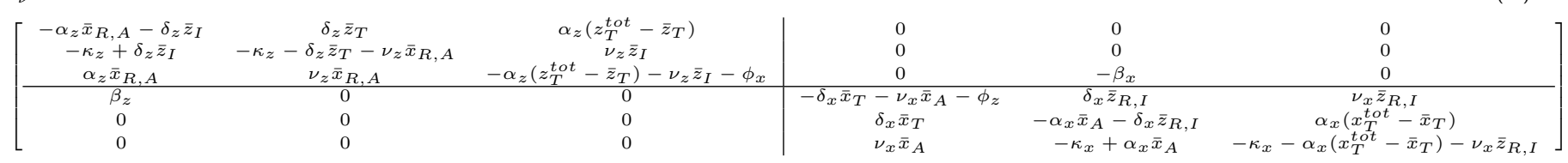




\subsubsection{Linear analysis}

As done earlier, we simplify the notation defining:

$$
\begin{aligned}
a_{1} & \doteq \delta_{z} \bar{z}_{T}, \\
b_{1} & \doteq \delta_{z} \bar{z}_{I}, \\
c_{1} & \doteq \alpha_{z} \bar{x}_{R, A}, \\
d_{1} & \doteq \alpha_{z}\left(z_{T}^{\text {tot }}-\bar{z}_{T}\right), \\
e_{1} & \doteq \kappa_{z}, \\
f_{1} & \doteq \nu_{z} \bar{x}_{R, A}, \\
g_{1} & \doteq \nu_{z} \bar{z}_{I}, \\
h_{1} & \doteq \phi_{x},
\end{aligned}
$$

$$
\begin{aligned}
a_{2} & \doteq \delta_{x} \bar{x}_{T} \\
b_{2} & \doteq \delta_{x} \bar{z}_{R, I}, \\
c_{2} & \doteq \alpha_{x} \bar{x}_{A}, \\
d_{2} & \doteq \alpha_{x}\left(x_{T}^{t o t}-\bar{x}_{T}\right) \\
e_{2} & \doteq \kappa_{x} \\
f_{2} & \doteq \nu_{x} \bar{x}_{A} \\
g_{2} & \doteq \nu_{x} \bar{z}_{R, I} \\
h_{2} & \doteq \phi_{z}
\end{aligned}
$$

Then, defining $\xi_{1}=\left[\begin{array}{lll}z_{T} & -z_{I} & -x_{R, A}\end{array}\right]^{\top}$ and $\xi_{2}=\left[\begin{array}{lll}z_{R, I} & -x_{T} & -x_{A}\end{array}\right]^{\top}$, the linearized system can be rewritten as the feedback interconnection of two linear systems:

$$
\dot{\xi}_{1}=A_{1} \xi_{1}+\beta_{x} B_{1} \omega_{2}, \quad \omega_{1}=C_{1} \xi_{1},
$$

and

$$
\dot{\xi}_{2}=A_{2} \xi_{2}+\beta_{z} B_{2} \omega_{1}, \quad \omega_{2}=C_{2} \xi_{2},
$$

where:

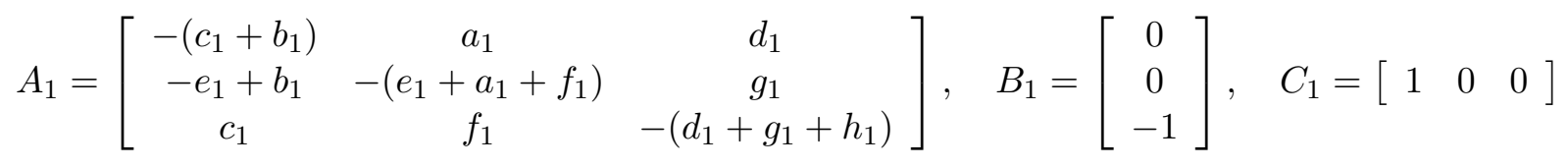

and

$$
A_{2}=\left[\begin{array}{ccc}
-\left(a_{2}+f_{2}+h_{2}\right) & b_{2} & g_{2} \\
a_{2} & -\left(c_{2}+b_{2}\right) & d_{2} \\
f_{2} & -e_{2}+c_{2} & -\left(e_{2}+d_{2}+g_{2}\right)
\end{array}\right], \quad B_{2}=\left[\begin{array}{l}
1 \\
0 \\
0
\end{array}\right], \quad C_{2}=\left[\begin{array}{lll}
0 & 1 & 0
\end{array}\right] .
$$

The transfer functions associated with the two subsystems (25) and (26) are:

$$
F_{1}(s)=-\frac{d_{1} s+a_{1} g_{1}+d_{1} e_{1}+d_{1} a_{1}+d_{1} f_{1}}{p_{1}(s)}
$$

and

$$
F_{2}(s)=\frac{a_{2} s+a_{2} e_{2}+a_{2} d_{2}+a_{2} g_{2}+d_{2} f_{2}}{p_{2}(s)},
$$

where $p_{1}(s)$ and $p_{2}(s)$ are third order polynomials having positive coefficients.

As in the case without titration reactions, the interconnection of the two subsystems is a negative feedback loop. The polynomials of the numerator and denominator of both the transfer functions have positive coefficients. As a consequence, the closed-loop characteristic polynomial has positive coefficients. A polynomial with positive coefficients cannot have nonnegative real roots. Therefore, instability can occur only with complex conjugate poles with positive real part, thus it can only be oscillatory. This confirms the result of our previous structural analysis.

Proposition 16. Consider system (15)-(20), where $\nu_{x}>0, \nu_{z}>0$, linearized around its only equlibrium point. Its characteristic polynomial has no real nonnegative roots. Instability can only occur due to complex roots with positive real part. 


\subsection{Numerical simulations}

\subsubsection{Randomized parameter search}

Table S1: Nominal parameters for the oscillator model (15)-(20)

\begin{tabular}{ll|ll} 
Rate & Value & Rate & Value \\
\hline$\alpha_{z}(/ \mathrm{M} / \mathrm{s})$ & $75 \cdot 10^{3}$ & $\alpha_{x}(/ \mathrm{M} / \mathrm{s})$ & $3 \cdot 10^{5}$ \\
$\delta_{z}(/ \mathrm{M} / \mathrm{s})$ & $3 \cdot 10^{5}$ & $\delta_{x}(/ \mathrm{M} / \mathrm{s})$ & $3 \cdot 10^{5}$ \\
$\nu_{z}(/ \mathrm{M} / \mathrm{s})$ & $3 \cdot 10^{5}$ & $\nu_{x}(/ \mathrm{M} / \mathrm{s})$ & $3 \cdot 10^{5}$ \\
$\beta_{z}(/ \mathrm{s})$ & $5 \cdot 10^{-3}$ & $\beta_{x}(/ \mathrm{s})$ & $2 \cdot 10^{-2}$ \\
$\kappa_{z}(/ \mathrm{s})$ & $1 \cdot 10^{-3}$ & $\kappa_{x}(/ \mathrm{s})$ & $1 \cdot 10^{-3}$ \\
$\phi_{z}(/ \mathrm{s})$ & $1 \cdot 10^{-3}$ & $\phi_{x}(/ \mathrm{s})$ & $1 \cdot 10^{-3}$ \\
$z_{T}^{\text {tot }}(\mathrm{nM})$ & 250 & $x_{T}^{\text {tot }}(\mathrm{nM})$ & 120 \\
$z_{I}^{\text {tot }}(\mathrm{nM})$ & 700 & $x_{A}^{\text {tot }}(\mathrm{nM})$ & 300
\end{tabular}

We numerically searched parameters that yield an oscillatory behavior in model (15)-(20). We generated random parameter values starting from the nominal parameter set listed on Table S1. We generated several hundreds of random parameter sets; reaction rates were varied in the range from $10^{-3}$ to $10^{3}$ times their nominal values; $z_{T}^{\text {tot }}, z_{I}^{\text {tot }}, x_{T}^{\text {tot }}$ and $x_{A}^{\text {tot }}$ were changed in the range from $10^{-1}$ to 10 times their nominal values. For each parameter set, the differential equations (15)-(20) are solved using the deterministic integrator RADAU, included in the software PyDSTool [4]. A parameter set is classified as "oscillatory" if at least 3 oscillations are detected, their average period is beween $0.5 h$ and $10 h$, and their average amplitude is larger than $10 \mathrm{nM}$. Each trajectory was integrated to have a duration of $20 \mathrm{~h}$. Our method follows the approach proposed in [5].

Period and amplitude were computed by identifying minima and maxima of oscillations, as shown in the inset of Fig. S3. For each three consecutive points of a trajectory, we define $d 1$ and $d 2$ as shown in Fig. S3 B: $d 1=p_{n}-p_{n-1}$ and $d 2=p_{n}-p_{n+1}$. If the product $d 1 \cdot d 2$ is positive and $d 1$ is positive, then $p_{n}$ is classified as a local maximum; if $d 1$ is negative, then $p_{n}$ is classified as a local minimum. Period and amplitude are computed from the identified maxima and minima, as sketched in Fig. S3 C. Period and amplitude are averaged over all the different measured peaks and wells and compared to the aforementioned thresholds.

Fig. S3 A shows the correlations among pairs of parameters that yield oscillations in the absence of direct titration reactions. Fig. S4 shows the results in the presence of titration reactions: the probability of oscillation is significantly increased. Some of the correlation plots show clear patterns. For example the plots clearly show that $z_{I}^{\text {tot }}$ should be larger than $z_{T}^{\text {tot }}$ and $x_{A}^{\text {tot }}$ larger than $x_{T}^{\text {tot }}$. Both $\beta_{z}$ and $\beta_{x}$ should be sufficiently large (relative to the nominal value).

\subsubsection{Classification of dynamic behaviors in a region of the parameter space}

We now classify equilibria as oscillatory or not by checking the eigenvalues of the Jacobian matrix (24) for a given parameter set (we recall that the system has a unique equilibrium for arbitrary choices of parameters). Starting from the nominal parameters listed in Table S1; all parameters were changed in the range from $10^{-1}$ to 10 times their nominal values. Two parameters were varied at a time, while others were held constant, to generate each subplot of Fig. S5 and S6. Equilibria were computed as the intersections of the analytical equilibrium expressions found at Section 2.2.1. Then, the stability properties of the equilibrium points are computed by finding the eigenvalues of the Jacobian evaluated at the equilibrium. When the Jacobian has at least one pair of complex conjugate eigenvalues with positive real part, it is classified as oscillatory; otherwise, it is classified as non-oscillatory and thus stable. 


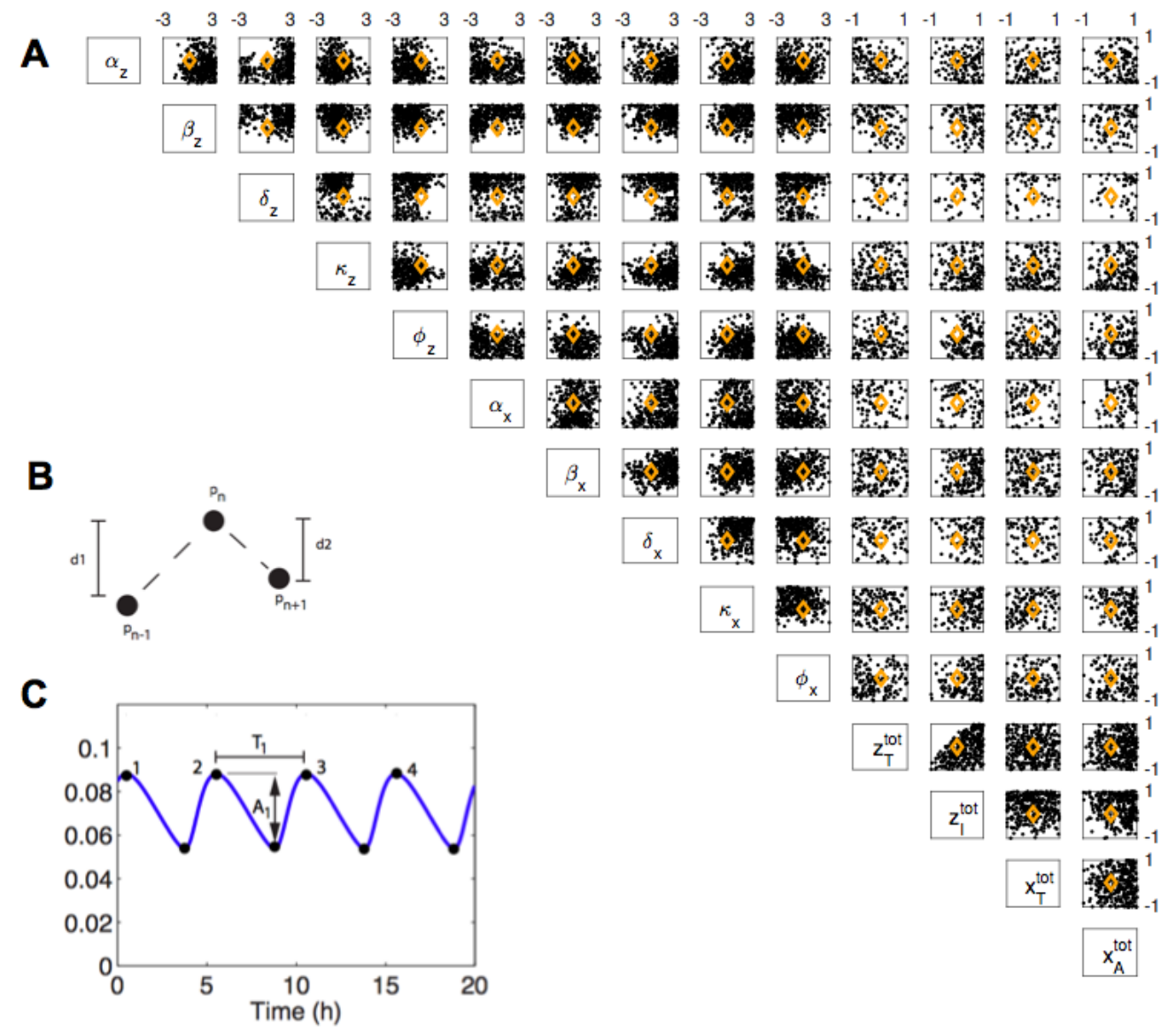

Figure S3: A: Absence of direct titration reactions. Correlation between randomly chosen parameters that yield oscillatory behavior. B: Points required for the identification of period and amplitude. C: Period and amplitude were measured as shown here and averaged over the trajectory. 


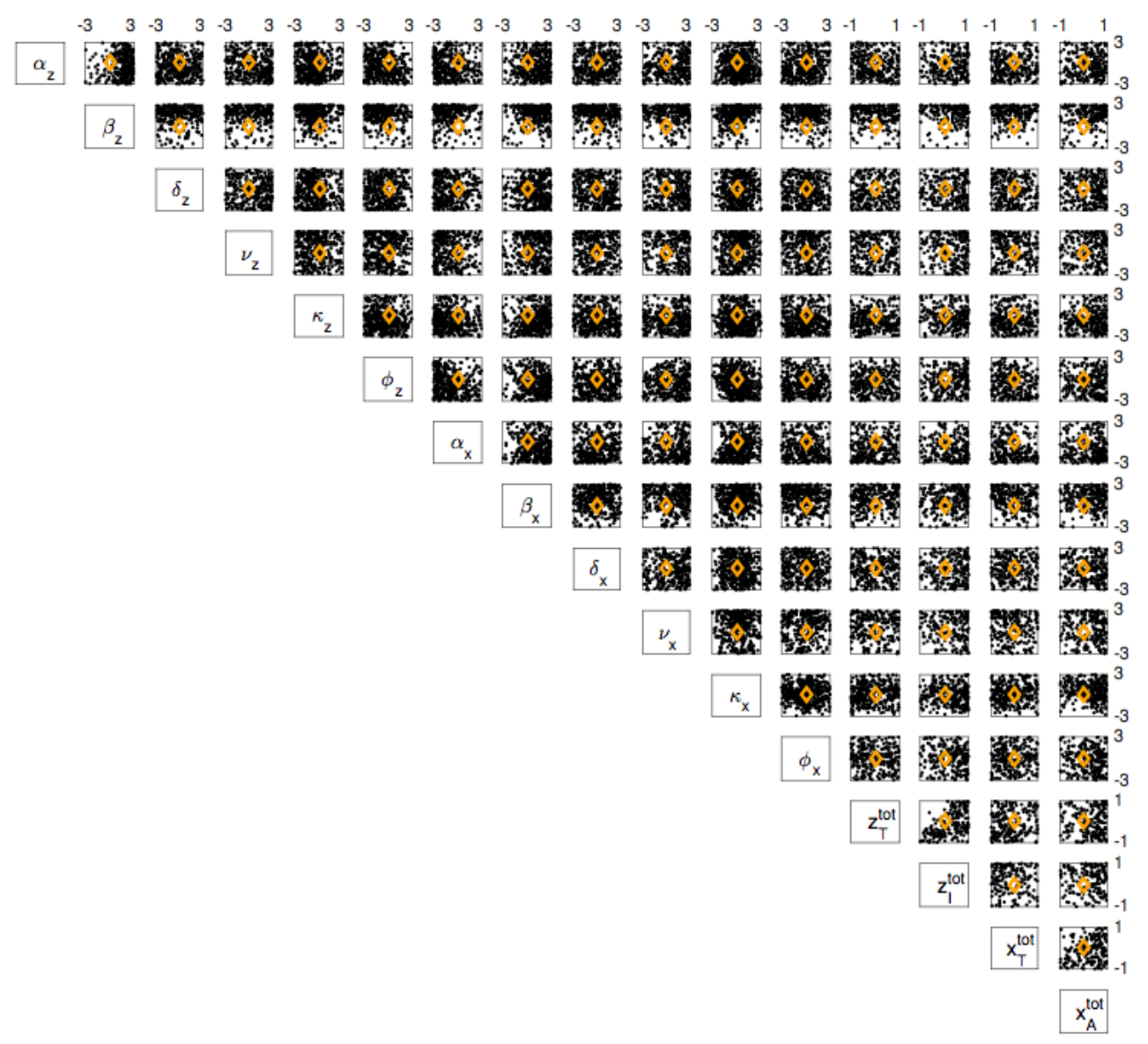

Figure S4: Presence of direct titration reactions. Correlation between randomly chosen parameters that yield oscillatory behavior. The probability of oscillation for a randomly chosen set of parameters significantly increases relative to Fig. S3. 
We summarize our results in Fig. S5 and S6, which show the influence of the parameters on the stability properties of the unique equilibrium of the system; we consider the case where titration reactions are absent (Fig. S5), and the case where titration reactions are comparable to the inhibition/activation rates of the regulators that interconnect the two modules (Fig. S6). The classification is color coded as follows: points where at least one pair of eigenvalues is complex with positive real part are shown in orange color; points at which we find real and negative eigenvalues or complex with negative real part are shown in blue color. These plots show some linear correlations among parameters that yield oscillations: $\left(\beta_{z}, x_{A}^{t o t}\right)$ and $\left(\beta_{x}, z_{I}^{t o t}\right)$ are positively correlated; $\left(\kappa_{x}, x_{A}^{t o t}\right)$ and $\left(\kappa_{z}, x_{I}^{t o t}\right)$ are negatively correlated in order to guarantee an oscillatory behavior. The presence of titration reactions considerably expands the oscillatory regions for all pairs of parameters.

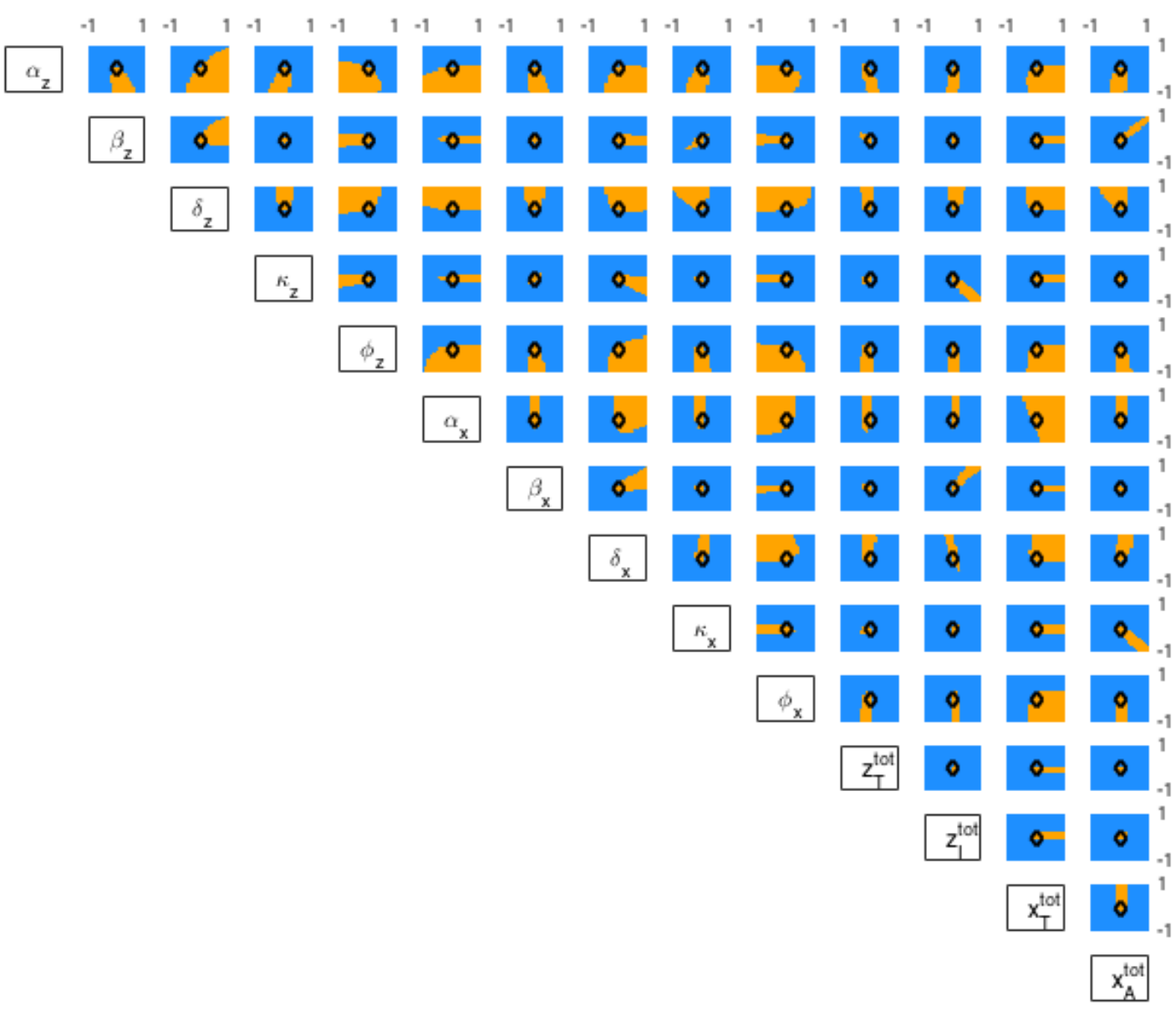

Figure S5: Absence of direct titration reactions: the log plots show the influence of variations of parameters on the stability of the equilibrium. Each parameter was varied between one tenth to ten times the nominal value (black diamond). Orange regions are oscillatory; blue regions indicate stable equilibria. 


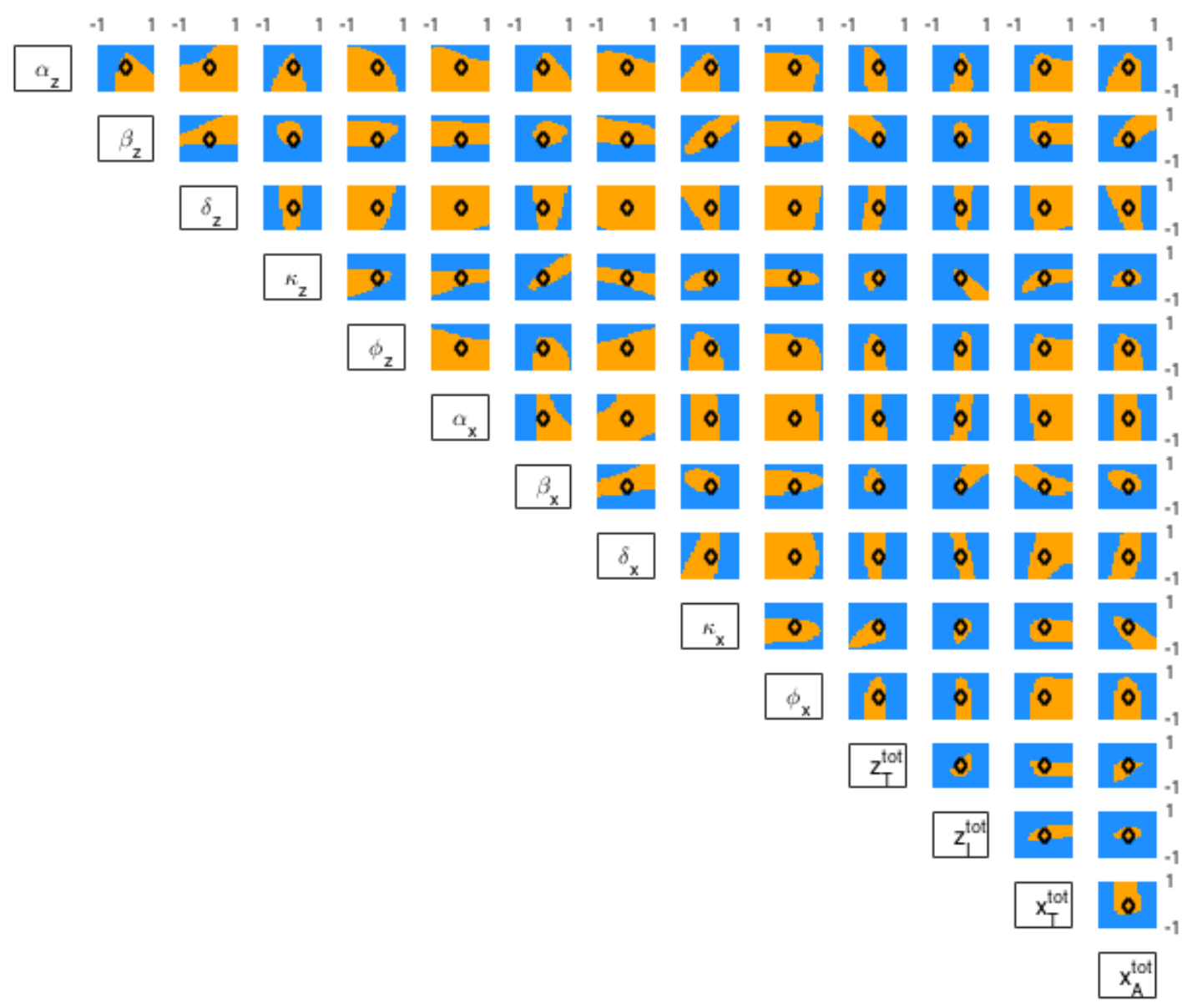

Figure S6: Presence of direct titration reactions: the log plots show the influence of variations of parameters on the stability of the equilibrium. Each parameter was varied between one tenth to ten times the nominal value (black diamond). Orange regions are oscillatory; blue regions indicate stable equilibria. The orange (oscillatory) regions are considerably larger than those in Fig. S5, where titration is absent. 


\section{Bistable system}

We build a bistable system via the feedback interconnection of two inhibited modules. The reactions describing the bistable system are:

\section{Subsystem 1}

Activation:

Output production:

Inhibition:

Direct titration:

Conversion:

Degradation:

$$
Z_{A}+Z_{T}^{*} \stackrel{\alpha_{z}}{\longrightarrow} Z_{T}
$$$$
Z_{T} \stackrel{\beta_{z}}{\longrightarrow} Z_{R, I}+Z_{T}
$$$$
X_{R, I}+Z_{T} \stackrel{\delta_{z}}{\longrightarrow} Z_{T}^{*}+Z_{A}^{*}
$$$$
X_{R, I}+Z_{A} \stackrel{\nu_{z}}{\longrightarrow} Z_{A}^{*}
$$$$
Z_{A}^{*} \stackrel{\kappa_{z}}{\longrightarrow} Z_{A}
$$$$
Z_{R, I} \stackrel{\phi_{z}}{\longrightarrow} 0
$$

\section{Subsystem 2}

$$
\begin{aligned}
& X_{A}+X_{T}^{*} \stackrel{\alpha_{x}}{\longrightarrow} X_{T} \\
& X_{T} \stackrel{\beta_{x}}{\longrightarrow} X_{R, I}+X_{T} \\
& Z_{R, I}+X_{T} \stackrel{\delta_{x}}{\longrightarrow} X_{T}^{*}+X_{A}^{*} \\
& Z_{R, I}+X_{A} \stackrel{\nu_{x}}{\longrightarrow} X_{A}^{*} \\
& X_{A}^{*} \stackrel{\kappa_{x}}{\longrightarrow} X_{A} \\
& X_{R, I} \stackrel{\phi_{x}}{\longrightarrow} 0
\end{aligned}
$$

The regulators interconnecting the modules are $x_{R, I}$ and $z_{R, I}$; both work as inhibitors. We assume mass conservation for species $Z_{T}, Z_{A}, X_{T}$, and $X_{A}: z_{T}^{\text {tot }}=z_{T}+z_{T}^{*}, z_{A}^{t o t}=z_{A}+z_{A}^{*}+z_{T}, x_{T}^{\text {tot }}=x_{T}+x_{T}^{*}, x_{A}^{t o t}=x_{A}+x_{A}^{*}+x_{T}$. The corresponding ODEs are:

$$
\begin{aligned}
\dot{z}_{T} & =\alpha_{z}\left(z_{T}^{\text {tot }}-z_{T}\right) z_{A}-\delta_{z} z_{T} x_{R, I}, \\
\dot{z}_{A} & =\kappa_{z}\left(z_{A}^{t o t}-z_{A}-z_{T}\right)-\alpha_{z}\left(z_{T}^{t o t}-z_{T}\right) z_{A}-\nu_{z} x_{R, I} z_{A}, \\
\dot{x}_{R, I} & =\beta_{x} x_{T}-\delta_{z} z_{T} x_{R, I}-\phi_{x} x_{R, I}-\nu_{z} x_{R, I} z_{A}, \\
\dot{x}_{T} & =\alpha_{x}\left(x_{T}^{t o t}-x_{T}\right) x_{A}-\delta_{x} x_{T} z_{R, I}, \\
\dot{x}_{A} & =\kappa_{x}\left(x_{A}^{t o t}-x_{A}-x_{T}\right)-\alpha_{x}\left(x_{T}^{t o t}-x_{T}\right) x_{A}-\nu_{x} x_{A} z_{R, I}, \\
\dot{z}_{R, I} & =\beta_{z} z_{T}-\delta_{x} x_{T} z_{R, I}-\phi_{z} z_{R, I}-\nu_{x} x_{A} z_{R, I} .
\end{aligned}
$$

Boxes highlight the terms corresponding to titration reactions. The two modules correspond to the subsystems $z_{T}-z_{A}-x_{R, I}$ and $x_{T}-x_{A}-z_{R, I}$.

Proposition 17. The solutions of the two separated modules, as well as those of the interconnected system (27)-(32), are globally bounded.

Proof. Analogous to the proofs of Propositions 5, 11 and 13.

\subsection{Analysis in the absence of direct titration reactions}

\subsubsection{Equilibrium conditions}

We consider system (27)-(32) in the absence of titration reactions, i.e., with $\nu_{x}=\nu_{z}=0$. We begin by setting equations $\dot{x}_{T}+\dot{x}_{A}=0$ and we combine them with $\dot{x}_{T}=0$. From here $\bar{z}_{R, I}$ is isolated as:

$$
\bar{z}_{R, I}=\frac{\alpha_{x}\left(x_{T}^{t o t}-\bar{x}_{T}\right) \bar{x}_{A}}{\delta_{x} \bar{x}_{T}}=\frac{\kappa_{x}\left(x_{A}^{t o t}-\bar{x}_{A}-\bar{x}_{T}\right)}{\delta_{x} \bar{x}_{T}} .
$$

We then find a relationship between $\bar{x}_{T}$ and $\bar{x}_{A}$ at steady state:

$$
\bar{x}_{A}=\frac{\kappa_{x}\left(x_{A}^{t o t}-\bar{x}_{T}\right)}{\alpha_{x}\left(x_{T}^{t o t}-\bar{x}_{T}\right)+\kappa_{x}} .
$$


Finally, setting equations $\dot{x}_{T}+\dot{x}_{A}=0$ and $\dot{z}_{R, I}=0$ we get:

$$
\bar{z}_{T}=\frac{\kappa_{x}}{\beta_{z}}\left(x_{A}^{t o t}-\bar{x}_{A}\left(\bar{x}_{T}\right)-\bar{x}_{T}\right)+\frac{\phi_{z}}{\beta_{z}} \bar{z}_{R, I}\left(\bar{x}_{T}\right) .
$$

The system is symmetric, so we now use the same procedure to obtain $\bar{x}_{T}\left(\bar{z}_{T}\right)$. We start setting $\dot{z}_{T}+\dot{z}_{A}=0$ and equation $\dot{x}_{R, I}=0$. We find:

$$
\bar{x}_{T}=\frac{\kappa_{z}}{\beta_{x}}\left(z_{A}^{t o t}-\bar{z}_{A}\left(\bar{z}_{T}\right)-\bar{z}_{T}\right)+\frac{\phi_{x}}{\beta_{x}} \bar{x}_{R, I}\left(\bar{z}_{T}\right) .
$$

Both equilibrium conditions are monotonically decreasing, which guarantees uniqueness of the equilibrium.

\subsubsection{Structural bistability}

The Jacobian matrix of system (27)-(32) with $\nu_{x}=\nu_{z}=0$ is:
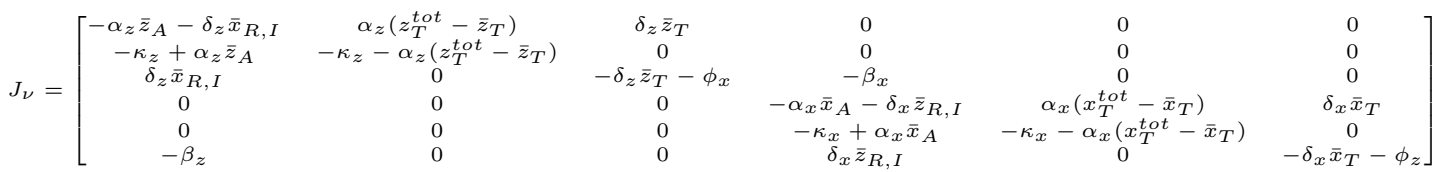

Here, the sign of the third and the sixth rows and columns has been changed (corresponding to a sign change for variables $x_{R, I}$ and $z_{R, I}$ ).

We say that a system is a strong candidate bistable system $[1,2]$ if it can become unstable exclusively due to a real eigenvalue that becomes positive.

Proposition 18. Under Assumptions 1, 2, 3, and 4, system (27)-(32) is a strong candidate bistable system.

Proof. We remind that, under our assumptions, $-\kappa_{z}+\alpha_{z} z_{A}>0$ and $-\kappa_{x}+\alpha_{x} x_{A}>0$. Then a similarity transformation $\hat{J}_{\nu}=T^{-1} J_{\nu} T$ can be applied, with $T=\operatorname{diag}\{-1,-1,-1,1,1,1\}$, such that $\hat{J}_{\nu}$ has non-negative off-diagonal entries, namely is a Metzler matrix, and negative diagonal entries. It is known (see for instance [3]) that a Metzler matrix has a real dominant eigenvalue: in this case, this means that an eigenvalue $\lambda_{1}$ exists such that $\operatorname{Re}\left(\lambda_{i}\right) \leq \lambda_{1}$, for $i=2,3, \ldots, 5$. Hence the proof follows.

The bistable nature of this system can be explained as follows. The transition to instability, if it happens, is due to a real eigenvalue which crosses the origin (0), becoming positive. This implies that the determinant of the matrix changes sign. Being the overall solution bounded, this implies that other two equilibria, both locally stable, necessarily appear (see $[1,2]$ for details).

\subsection{Analysis in the presence of direct titration reactions}

\subsubsection{Equilibrium conditions}

To derive $\bar{z}_{T}$ as a function of $\bar{x}_{T}$, we set equations $\dot{x}_{T}+\dot{x}_{A}=0$ and $\dot{x}_{T}=0$, and we find two different expressions for $\bar{z}_{R, I}$. Equating these expressions we obtain:

$$
\bar{z}_{R, I}=\frac{\alpha_{x}\left(x_{T}^{t o t}-\bar{x}_{T}\right) \bar{x}_{A}}{\delta_{x} \bar{x}_{T}}=\frac{\kappa_{x}\left(x_{A}^{t o t}-\bar{x}_{A}-\bar{x}_{T}\right)}{\delta_{x} \bar{x}_{T}+\nu_{x} \bar{x}_{A}},
$$


and we find a relationship between $\bar{x}_{T}$ and $\bar{x}_{A}$ at steady state. As done before, we derive the equilibrium of $\bar{x}_{A}$ as the solution of the second order equation $a_{x} \bar{x}_{A}^{2}+b_{x} \bar{x}_{A}+c_{x}$, where $a_{x}=\left(\frac{\alpha_{x} \nu_{x}}{\delta_{x}}\right) \frac{x_{T}^{\text {tot }}-\bar{x}_{T}}{\bar{x}_{T}}, b_{x}=\left(\alpha_{x}\left(x_{T}^{\text {tot }}-\bar{x}_{T}\right)+\kappa_{x}\right)$ and $c_{x}=-\kappa_{x}\left(x_{A}^{t o t}-\bar{x}_{T}\right)$. Assuming $x_{A}^{t o t}>x_{T}^{t o t}$, since $a_{x} c_{x}<0$, the only admissible positive solution is:

$$
\bar{x}_{A}\left(\bar{x}_{T}\right)=\frac{-b_{x}+\sqrt{b_{x}^{2}-4 a_{x} c_{x}}}{2 a_{x}} .
$$

Finally, setting equations $\dot{x}_{T}+\dot{x}_{A}=0$ and $\dot{z}_{R, I}=0$ we get:

$$
\bar{z}_{T}=\frac{\kappa_{x}}{\beta_{z}}\left(x_{A}^{t o t}-\bar{x}_{A}\left(\bar{x}_{T}\right)-\bar{x}_{T}\right)+\frac{\phi_{z}}{\beta_{z}} \bar{z}_{R, I}\left(\bar{x}_{T}\right) .
$$

With a similar procedure we can get the equilibrium condition for $\bar{x}_{T}\left(\bar{z}_{T}\right)$ and the remaining equilibria.

Once we find the admissible equilibrium values $\bar{z}_{T}, \bar{z}_{A}, \bar{x}_{T}$ and $\bar{x}_{A}$ we can find $\bar{z}_{R, I}$ and $\bar{x}_{R, I}$.

$$
\begin{aligned}
& \dot{x}_{R, I}=0 \quad \Longrightarrow \quad \bar{z}_{R, I}=\frac{\kappa_{x}\left(x_{A}^{t o t}-\bar{x}_{A}-\bar{x}_{T}\right)}{\delta_{x} \bar{x}_{T}+\nu_{x} \bar{x}_{A}}, \\
& \dot{z}_{R, I}=0 \quad \Longrightarrow \quad \bar{x}_{R, I}=\frac{\kappa_{z}\left(z_{A}^{t o t}-\bar{z}_{A}-\bar{z}_{T}\right)}{\delta_{z} \bar{z}_{T}+\nu_{z} \bar{z}_{A}} .
\end{aligned}
$$

\subsubsection{Structural bistability}

In the presence of direct titration reactions the Jacobian becomes:

$$
J_{\nu}=
$$
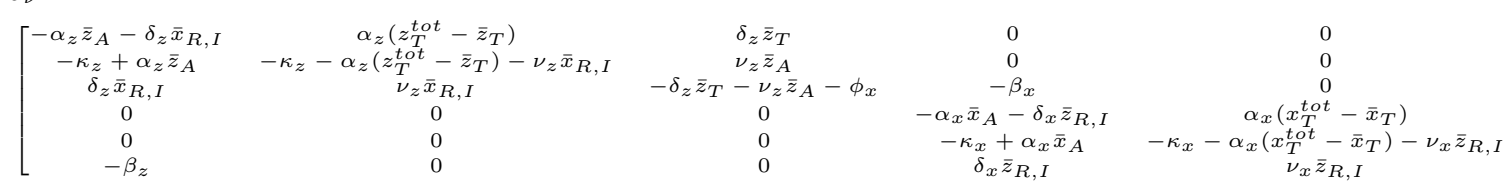

(37)

As done earlier, the sign of the third and the sixth rows and columns has been changed (corresponding to a sign change for variables $x_{R, I}$ and $z_{R, I}$ ).

In the presence of direct titration reactions it is more difficult to formally show that the feedback of the two subsystems is a candidate bistable system. We can however note that:

a) For "small" $\nu_{x}$ and $\nu_{z}$, the two subsystems are "almost" in the same condition of Proposition 18, hence bistability occurs. Also, if we can assume that $\bar{z}_{A}>\kappa_{z} / \alpha_{z}$ and $\bar{x}_{A}>\kappa_{x} / \alpha_{x}$, then the same considerations as in the case of no titration apply and a bistable behavior is expected.

b) More than one equilibrium point may appear for suitable choice of the parameters. When three equilibria appear, if the Jacobian is invertible, then necessarily one of the equilibria is unstable with a real positive unstable eigenvalues; this can be explained with the so called degree theory; we refer the reader to reference [1] for additional details. The other two equilibria are expected to be stable.

c) Numerical simulations on a wide range of parameters confirm that this system can be bistable.

\subsection{Numerical simulations}

\subsubsection{Probability of bistable behavior}

As done for the oscillator, we explored the probability of obtaining a bistable behavior for random choices of the parameters around the nominal set in Table S2. The reaction rate parameters were randomly selected in the range from $10^{-2}$ to $10^{2}$ their nominal value; parameters $z_{T}^{\text {tot }}, x_{A}^{t o t}, x_{T}^{\text {tot }}, x_{A}^{\text {tot }}$ were instead varied between one tenth and 
ten times their nominal value. A set of parameters is classified as bistable if a) three equilibria are identified by numerically finding the intersections of the equilibrium conditions derived earlier, and b) two of these equilibria are stable (all eigenvalues have negative real part) and one is unstable (at least one eigenvalue has positive real part). Fig. S7 and S8 show that the system can exhibit bistability in a wide range of parameters, however large titration reaction rates (Fig. S8) significantly increase the probability of bistable behavior.

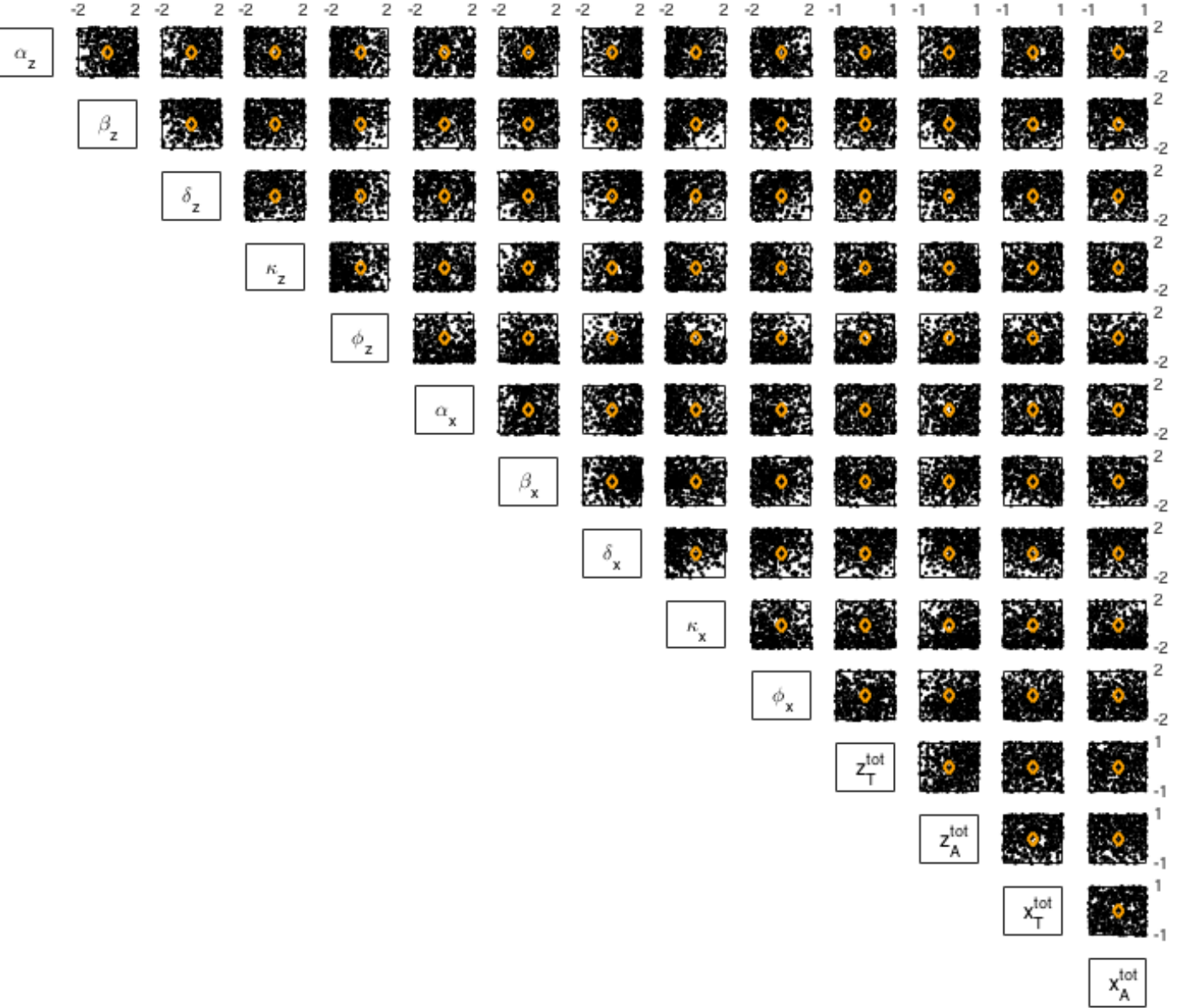

Figure S7: Absence of direct titration reactions. Log plot showing the correlation between randomly chosen parameters that yield bistable behavior. Nominal parameters (Table S2) are shown in the orange diamond; reaction rate parameters were varied between a factor $10^{-2}$ and $10^{2}$ of their nominal value (with $\nu=0$ ), while total concentrations were varied between one tenth and ten times their nominal value.

\subsubsection{Bistable behavior in a region of the parameter space}

We use parameters listed in Table S2 to explore numerically the bistability regions. As done for the randomized parameter classification, a parameter set yields a bistable behavior if three equilibria, two stable and one unstable, 


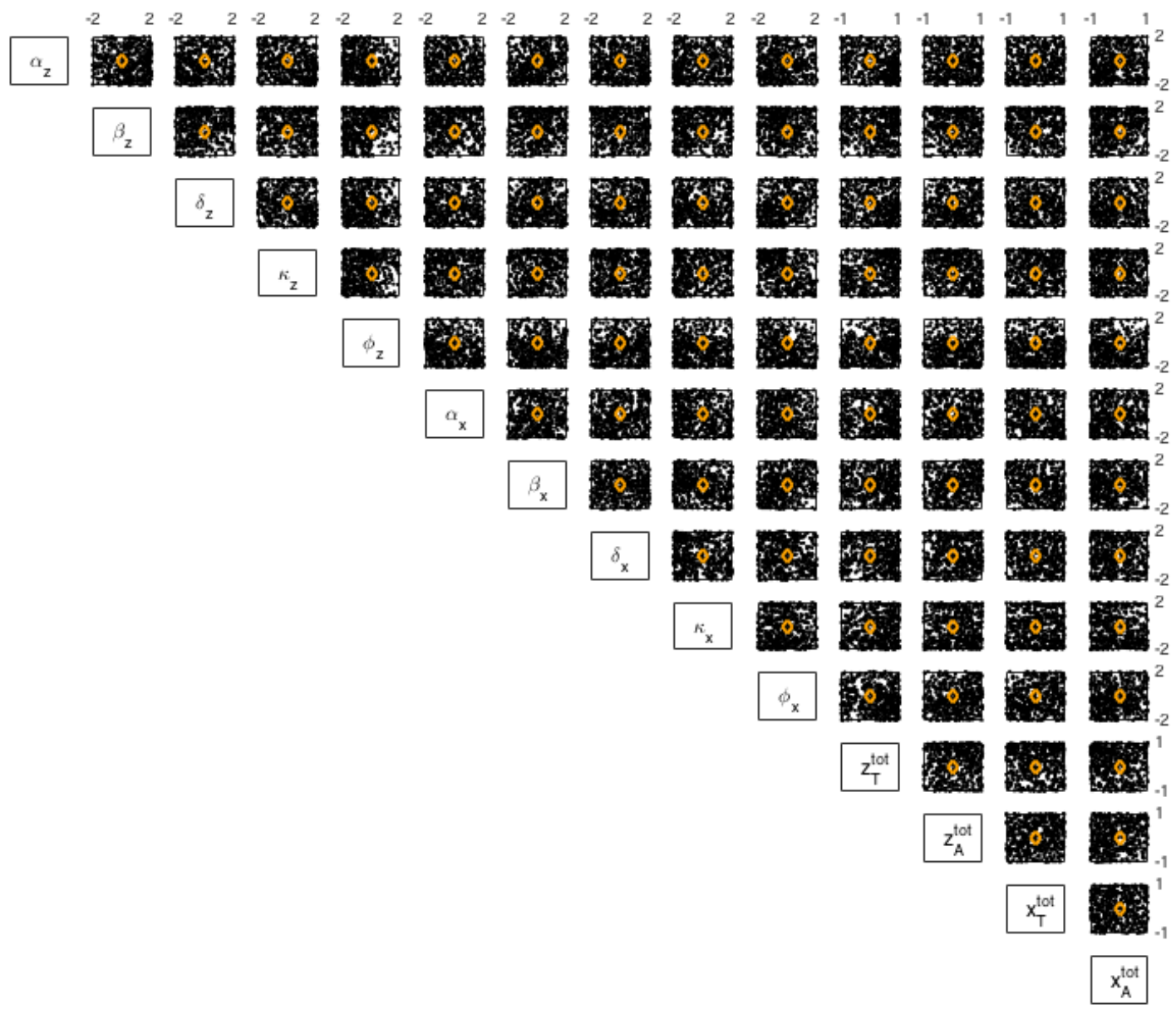

Figure S8: Presence of direct titration reactions. Log plot showing the correlation between randomly chosen parameters that yield bistable behavior. Nominal parameters (Table S2) are shown in the orange diamond; parameters were varied between a factor $10^{-2}$ and $10^{2}$ of their nominal value.

Table S2: Nominal parameters for the bistable circuit

\begin{tabular}{lll} 
Units: $[\mathrm{nM}]$ & Units: $[1 / \mathrm{s}]$ & Units: $[1 / \mathrm{M} / \mathrm{s}]$ \\
\hline$z_{T}^{\text {tot }}=100$ & $\beta_{z}=0.0021$ & $\alpha_{z}=3 \times 10^{4}$ \\
\hline$x_{T}^{\text {tot }}=100$ & $\beta_{x}=\beta_{z}$ & $\alpha_{x}=\alpha_{z}$ \\
\hline$z_{A}^{\text {tot }}=200$ & $\kappa_{z}=3 \times 10^{-4}$ & $\delta_{z}=3 \times 10^{4}$ \\
\hline$x_{A}^{\text {tot }}=200$ & $\kappa_{x}=\kappa_{z}$ & $\delta_{x}=\delta_{z}$ \\
\hline & $\phi_{z}=0.001$ & $\nu_{z}=\delta_{z}$ \\
\hline & $\phi_{x}=\phi_{z}$ & $\nu_{x}=\nu_{z}$ \\
\hline
\end{tabular}


are identified. Here, we vary only two parameters at a time, keeping the others fixed at their nominal value. In Fig. S9 and S10 we show the bistability domains (orange regions), in the absence of direct titration reactions (Fig. S9) and in the presence of titration (Fig. S10).

In the absence of titration reactions there are many pairs of parameters where the bistability region is very narrow. This makes the system less robust over the parameter space since any change in the parameters will cause the system to lose bistability. It also shows that there is a linear correlation in many pairs of parameters for a bistable behavior: $\left(\beta_{z}, \beta_{x}\right),\left(\kappa_{z}, \kappa_{x}\right),\left(\kappa_{x}, z_{A}^{t o t}\right),\left(\beta_{z}, x_{A}^{t o t}\right),\left(\kappa_{z}, x_{A}^{t o t}\right),\left(\delta_{x}, \phi_{z}\right)$ and $\left(\delta_{z}, \phi_{x}\right)$ show a positive correlation, while $\left(\beta_{z}, \kappa_{z}\right),\left(\beta_{z}, z_{A}^{\text {tot }}\right),\left(\kappa_{z}, z_{A}^{\text {tot }}\right),\left(\beta_{x}, x_{A}^{\text {tot }}\right)$ and $\left(\kappa_{x}, x_{A}^{\text {tot }}\right)$ show a negative correlation to present bistable behavior. Fig. S10 clearly shows that all the regions of bistability are expanded when the titration reaction are present.

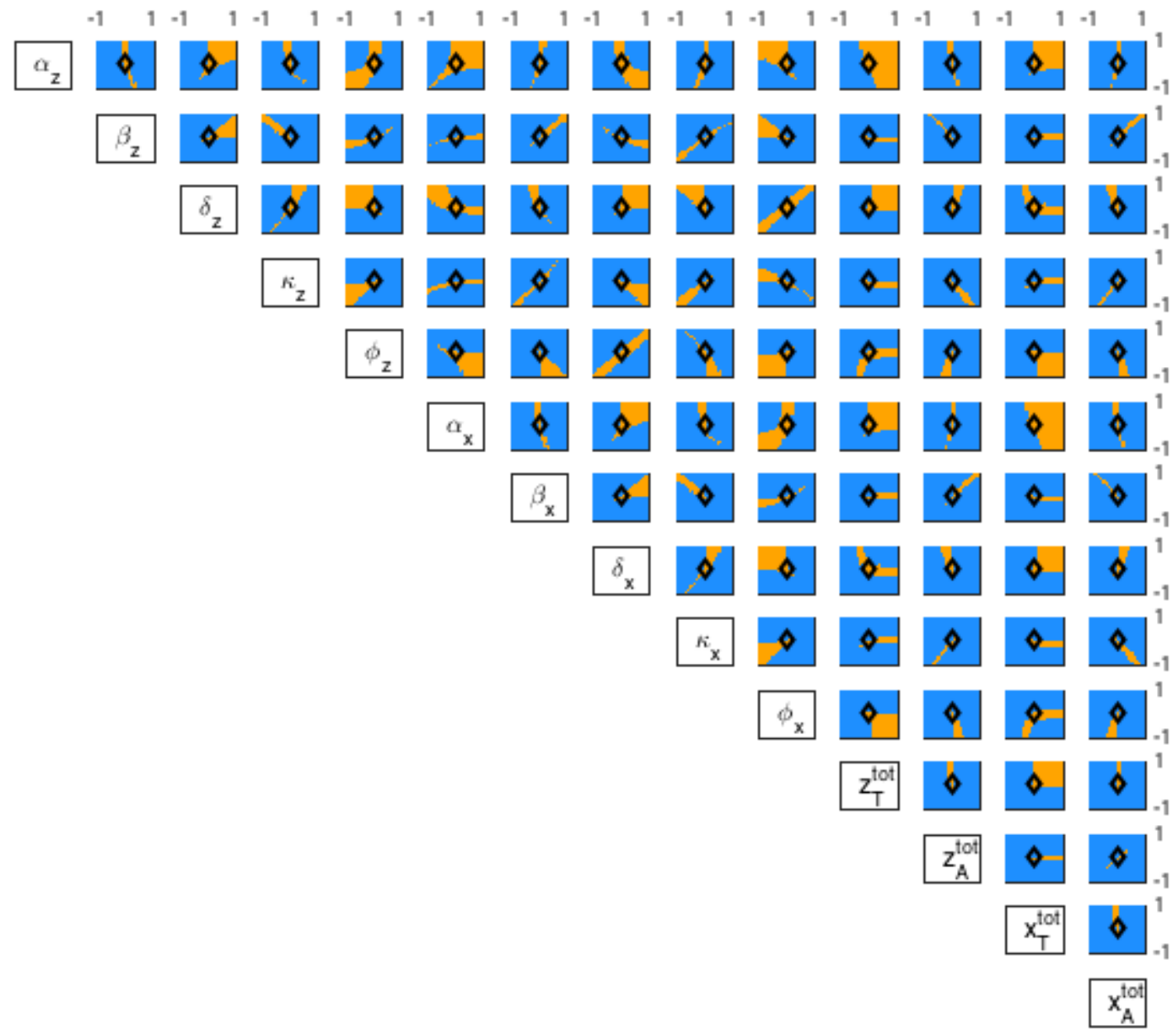

Figure S9: Absence of direct titration reactions. Axes are in log scale. The orange areas are bistable regions. Blue areas correspond to a unique stable steady state. Nominal parameters are shown as a black diamond. 


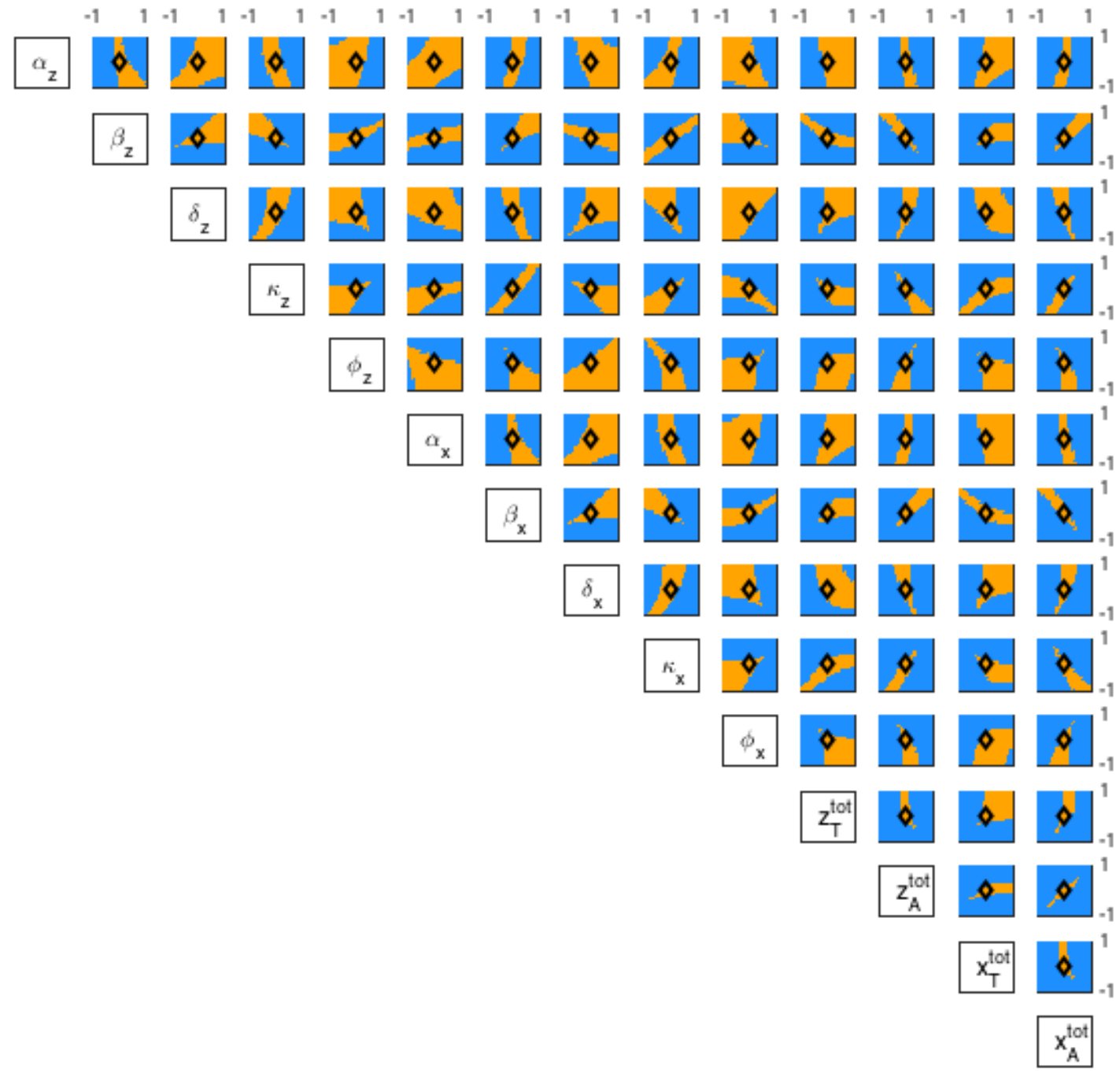

Figure S10: Presence of direct titration reactions. Axes are in log scale. The orange areas are bistable regions, which are clearly expanded relative to Fig. S9. Blue areas correspond to a unique stable steady state. Nominal parameters are shown as a black diamond 


\section{References}

[1] F. Blanchini, E. Franco, and G. Giordano. A structural classification of candidate oscillatory and multistationary biochemical systems. Bulletin of Mathematical Biology, 76(10):2542-2569, 2014.

[2] F. Blanchini, E. Franco, and G. Giordano. Structural conditions for oscillations and multistationarity in aggregate monotone systems. In Proceedings of the 54th IEEE Conference on Decision and Control, pages 609-614, 2015.

[3] F. Blanchini and S. Miani. Set-theoretic methods in control. Systems \& Control: Foundations \& Applications. Birkhäuser, Basel, 2015.

[4] R. Clewley, W. Sherwood, M. LaMar, and J. Guckenheimer. PyDSTool, a software environment for dynamical systems modeling. 2007.

[5] C. E. Cuba, A. R. Valle, G. Ayala-Charca, E. R. Villota, and A. M. Coronado. Influence of parameter values on the oscillation sensitivities of two p53-Mdm2 models. Systems and Synthetic Biology, 9(3):77-84, 2015.

[6] H. K. Khalil. Nonlinear Systems. Prentice Hall, 2002. 